\title{
Pattern formation in auxin flux
}

\author{
C. Feller • J. P. Gabriel • C. Mazza • F. Yerly
}

Received: 12 October 2011 / Revised: 22 January 2013 / Published online: 23 February 2013

(C) Springer-Verlag Berlin Heidelberg 2013

\begin{abstract}
The plant hormone auxin is fundamental for plant growth, and its spatial distribution in plant tissues is critical for plant morphogenesis. We consider a leading model of the polar auxin flux, and study in full detail the stability of the possible equilibrium configurations. We show that the critical states of the auxin transport process are composed of basic building blocks, which are isolated in a background of auxin depleted cells, and are not geometrically regular in general. The same model was considered recently through a continuous limit and a coupling to the von Karman equations, to model the interplay of biochemistry and mechanics during plant growth. Our conclusions might be of interest in this setting, since, for example, we establish the existence of Lyapunov functions for the auxin flux, proving in this way the convergence of pure transport processes toward the set of equilibrium points.
\end{abstract}

Keywords Auxin flux $\cdot$ Pattern formation $\cdot$ Reinforced random walks

C. Feller · J. P. Gabriel · C. Mazza $(\varangle) \cdot$ F. Yerly

Department of Mathematics, University of Fribourg,

Ch. du Musée 23, 1700 Fribourg, Switzerland

e-mail: christian.mazza@unifr.ch

C. Feller

e-mail: chrystel.feller@unifr.ch

J. P. Gabriel

e-mail: jean-pierre.gabriel@unifr.ch

F. Yerly

e-mail: florence.yerly@unifr.ch

C. Feller $\cdot$ C. Mazza $\cdot$ F. Yerly

Swiss Institute of Bioinformatics,

Quartier Sorge, Bâtiment Génopode, 1015 Lausanne, Switzerland 


\section{Introduction}

The plant hormone auxin plays a fundamental role in plant development (Reinhardt et al. 2000, 2003), and its spatial distribution in plants tissues is critical for plant morphogenesis. Auxin accumulation is spatially localized in specific set of cells, where it induces the emergence of new primordia (Reinhardt et al. 2000). A fundamental problem consists in understanding how such auxin maxima appear, and how they induce the regular pattern observed in plants (see e.g. Hamant and Traas 2009). Direct quantitative measurements of auxin distribution in plant tissues are very difficult due to the small size of the meristematic tissues at the time of patterning. Therefore, biologists rely on indirect markers based on auxin-regulated genes that encode fluorescent proteins. Figure 1 shows a typical output, where domains rich in auxin appear as regions of strong yellow fluorescence.

On the other hand, experiments show that phyllotaxis strongly depends on the plant physical properties, more precisely on elasticity (Green 1980; Dumais and Steele 2000; Dumais 2007), and physical forces provide information for plant patterning (Hamant and Traas 2009). Basically, turgor pressure induces stress, which is related to the associated deformation or strain through Young constants: see e.g. Boudaoud (2010) where these notions are explained in the context of plant growth. Experiments have shown that lowering the stiffness of cell walls in the meristem leads to the emergence of new primordia (Hamant et al. 2008). However, the interactions between physics-based and biochemical control of phyllotaxis is still poorly understood.

Recently, new biologically plausible mathematical models of auxin transport have been proposed (Barbier de Reuille et al. 2006; Heisler 2006; Jönsson et al. 2006; Smith et al. 2006), each of them being able to reproduce some aspects of phyllotaxis in simulations. New mathematical models were also proposed for the plant mechanics (Mjolsness 2006), and for the interaction between mechanics and biochemistry (Shipman and Newell 2005; Newell et al. 2008). In the latter, the authors use the model for the polar auxin flux proposed in Jönsson et al. (2006) for modelling the stress field in their mechanical model. All these models are based on hypotheses that have not been verified experimentally; however, they provide new scenarios for understanding plant growth that can be tested experimentally.

Fig. 1 Inflorescence shoot apical meristem of Arabidopsis thaliana. Zones with high auxin concentration are highlighted by the fluorescent yellow signal auxin reporter from DR5::YFP. The red signal is highlighting cell walls stain, using propidium iodide (color figure online)

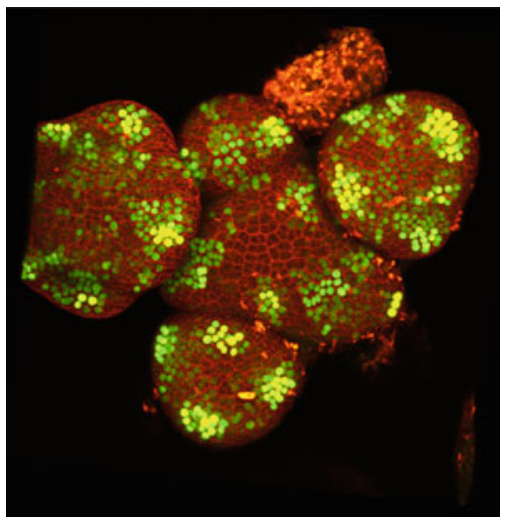


In the following, we consider the models in Jönsson et al. (2006) and Smith et al. (2006), based on polar auxin flux. Polar auxin flux (or anisotropic flux) results from uneven accumulation of the auxin transport regulator PIN in cell membranes. An essential component is a positive feedback between auxin flux and PIN localization, resulting in the reinforcement of polar auxin transport to dedicated routes which develop into vascular tissues. We will not enter here into these considerations, but focus on simple models of transport processes (see e.g. the discussion in Jönsson et al. 2006 and Shipman and Newell 2005), where a quasi-equilibrium is assumed for PIN proteins. The molecules present in some cell $i$ may be transported to any neighbouring cell $j$, but they are preferentially transported to the neighbours with the highest auxin concentrations.

Traditionally, models of patterning and morphogenesis have used reaction-diffusion theory. Turing demonstrated how, under some hypotheses, the regular patterns observed in phyllotaxis can be predicted (Turing 1952). He showed that a combination of diffusion and a chemical reaction could give rise to regular patterns. Interesting models are described in Meinhardt (1982) and Thornley (1975) which can, under some hypotheses, predict phyllotactic patterns. As stated previously, the auxin flux is strongly polarized, a phenomenon that cannot be described with reactiondiffusion models. The recent mathematical models given in Barbier de Reuille et al. (2006), Jönsson et al. (2006), Smith et al. (2006) are based on transport processes. We propose here a mathematical study of related dynamical systems. We focus on their equilibrium points and analyse their geometrical structure and stability.

Besides stable auxin peaks, the model generates intervening areas of auxin depletion, as it is observed experimentally. These auxin depleted sites reflect an indirect repulsion mechanism since auxin molecules diffusing through the tissue will be attracted to the peaks, and diverted from the depleted areas. This idea of repulsion or spacing mechanism was already considered a long time ago (Hofmeister 1868). Many authors have used this hypothesis to develop very interesting mathematical models, all leading to phyllotactic patterns observed in nature, like Fibonacci numbers, the Golden Angle or helical lattices, see Adler (1974), Atela et al. (2002), Douady and Couder (1996), Kunz (1995), Levitov (1991).

\section{Model}

The auxin flux is present everywhere in the plant, so that we choose to describe the various plant cells as a connected graph $(\Lambda, E)$. The node set $\Lambda$ represents the cells and $E$ the set of edges. Any edge $e=(i \rightarrow j), i, j \in \Lambda$ indicates that some auxin molecule can move from cell $i$ to cell $j$. This graph is undirected, and we write $i \sim j$ to denote that cells $i$ and $j$ are nearest neighbours, so that auxin can move from cell $i$ to cell $j$, at some rate $q_{i j}$. These transition rates are not well understood at present time and one must rely on simple models. They should capture the fact that an auxin molecule present in some cell $i$ has the tendency to move to a cell $j \sim i$ when the concentration $a_{j}$ of auxin molecules present in cell $j$ is high. The simplest model accounting for this idea is given by Jönsson et al. (2006) 


$$
q_{i j}=\frac{a_{j}}{\kappa+\sum_{k \sim i} a_{k}},
$$

for some positive constant $\kappa$, which is of Michaelis-Menten or Monod type. Let $L=$ $|\Lambda|$ be the number of cells. In the model given in Jönsson et al. (2006) (see also Smith et al. 2006; Sahlin et al. 2009), $a_{i}(t)$, for $i \in \Lambda$, denotes the concentration of auxin molecules in cell $i$ at time $t$, and is assumed to evolve according to the differential equations

$$
\begin{aligned}
\frac{\mathrm{d} a_{i}}{\mathrm{dt}} & =D \sum_{k \sim i}\left(a_{k}-a_{i}\right)+T \sum_{k \sim i}(a_{k} \underbrace{\frac{a_{i}}{\kappa+\sum_{j \sim k} a_{j}}}_{=q_{k i}(\boldsymbol{a})}-a_{i} \underbrace{\frac{a_{k}}{\kappa+\sum_{j \sim i} a_{j}}}_{=q_{i k}(\boldsymbol{a})}) \\
& =: f_{i}(\boldsymbol{a})
\end{aligned}
$$

for $i=1, \ldots, L$. The terms $D a_{i}+T a_{i} q_{i k}$ give the mean number of auxin molecules moving from cell $i$ to cell $k$ per unit time. The first part of the right member in (2), $D \sum_{k \sim i}\left(a_{k}-a_{i}\right)$, is a diffusive part, usually assumed to be weak with a small diffusion coefficient $D$. The second term corresponds to the mass transport process, which is known to be the main actor of the patterning process in plants. One can add auxin production and degradation terms, but, there is no clear biological evidence about where auxin is produced, and experiments show that it is not produced in the meristem, but imported from the leaves (Reinhardt et al. 2000; Reinhardt 2005).

The system (2) can be written in the compact form

$$
\frac{\mathrm{d} \boldsymbol{a}}{\mathrm{dt}}=f(\boldsymbol{a}),
$$

where $\boldsymbol{a}(t)=\left(a_{i}(t)\right)_{1 \leq i \leq L}$ is the vector of auxin concentrations. The related equilibrium points are the vectors $\overline{\boldsymbol{a}}$ satisfying $f(\overline{\boldsymbol{a}})=0$. They are the candidates for describing the equilibrium auxin concentrations.

The equilibrium points play a fundamental role in the dynamics, and one can suspect that any solution $\boldsymbol{a}(t)$ of (2) will approach such equilibrium points as $t$ is large. Of course, this is wrong for general dynamical systems, but here, the model is supposed to catch pieces of biological reality, and the robustness of the regular geometries observed in plants suggests that this might well be the case. Some of these equilibrium points are repulsive or unstable, that is, the orbits or the solutions of (2) will avoid them. In the contrary, some of them will be attractive. Given an equilibrium point $\overline{\boldsymbol{a}}$, a mathematical way of checking the stability or the unstability of $\overline{\boldsymbol{a}}$ is to compute the Jacobian $\mathrm{d} f(\overline{\boldsymbol{a}})$, by retaining only its spectrum, that is the set of all eigenvalues of $\mathrm{d} f(\overline{\boldsymbol{a}})$.

Definition 1 An equilibrium point $\boldsymbol{a}$ is called linearly stable when all the eigenvalues of the Jacobian evaluated at $\boldsymbol{a}$ have non-positive real parts. For the remainder of the article, we use the abbreviated term $l$-stable.

An equilibrium point $\boldsymbol{a}$ is unstable when there is an eigenvalue having a positive real part. 
In the next section, we will check the existence of a solution of system (2) and give some properties (non-negativity and conservativity).

Section 4 is concerned with the characterization of the set of equilibrium points, mainly focusing on pure transport processes (i.e. the diffusive part is set to zero, $D=$ 0 ). We then study the stability of the obtained equilibrium points by using the Jacobian. We present various results on graphs of interest for plant patterning questions, like the circle or the two-dimensional grid. Section 6 considers the asymptotic behaviour of the solutions of (2) and establish in Proposition 1 that every solution converges toward the set of equilibrium points. Our technique is based on Lyapunov functions, that is, we look for a function which should be decreasing along the orbits of (2).

Finally, the model provides an interesting conclusion: for most graphs, $l$-stable configuration are composed of building blocks isolated in a sea of auxin depleted cells. This might be the basis for repulsion between primordia: auxin molecules will not have the tendency to move toward them, leading to indirect repulsion.

\section{Basic properties of the auxin flux}

Proposition 1 For every initial conditions in $\mathbb{R}_{>0}^{L}$, system (2) admits an unique solution $\boldsymbol{a}(t)$ defined over $[0,+\infty)$. Moreover the solution $\boldsymbol{a}(t)$ is non-negative and conservative, i.e.

$$
\forall t \in \mathbb{R}_{\geq 0}, \quad \sum_{i}^{L} a_{i}(t)=\sum_{i}^{L} a_{i}(0)
$$

Furthermore, if $a_{i}(0)$ is strictly positive, then $a_{i}(t)$ remains strictly positive for all $t>0$.

For pure transport processes (i.e. $D=0$ ), an auxin depleted cell $i\left(\right.$ i.e. $\left.a_{i}(0)=0\right)$ remains depleted i.e. $a_{i}(t) \equiv 0, \forall t>0$.

Proof See Appendix A.

Proposition 2 If the graph is connected and $D>0$, the only equilibrium point of (2) in $\mathbb{R}_{+}^{L}$ admitting zero components is the origin.

Proof Let us rewrite the system (2), for $1 \leq i \leq L$

$$
\dot{a}_{i}=D \sum_{k \sim i} a_{k}+T \sum_{k \sim i}\left(\frac{a_{k}}{\kappa+\sum_{j \sim k} a_{j}}-\frac{a_{k}}{\kappa+\sum_{j \sim i} a_{j}}-\frac{D}{T}\right) a_{i}
$$

with the initial condition $\boldsymbol{a}(0) \in \mathbb{R}_{+}^{L}$.

Assume that the $i$ th component of an equilibrium point $\boldsymbol{a} \in \mathbb{R}_{+}^{L}$ of (3) is zero i.e. $a_{i}=0$. Clearly (3) entails $\sum_{k \sim i} a_{k}=0$ and the non-negativity of each term, $a_{k}=0$ for all $k \sim i$. Since the graph is connected we deduce that $a_{k}=0$ for all $1 \leq k \leq L$. 


\section{Characterization of the equilibrium points}

Our first aim is to investigate the equilibrium points of system (2), i.e. the elements $\boldsymbol{a} \in \mathbb{R}_{\geq 0}^{L}$ solving the equations $f(\boldsymbol{a})=0$.

We will use notions from continuous time Markov chain theory, and hence consider generator matrices $Q: \Lambda \times \Lambda \longrightarrow \mathbb{R}, Q=\left\{q_{i j}, i, j \in \Lambda\right\}$, for (continuous time) Markov chain on $\Lambda$. Theses matrices have the properties

$$
q_{i j} \geq 0, \quad \text { for } i \neq j \text { and } q_{i i}=-\sum_{j \neq i} q_{i j}
$$

The auxin flux described by (2) contains implicitly a generator matrix $\boldsymbol{Q}(D, T, \boldsymbol{a})$ given by

$$
\begin{cases}q_{i j}(D, T, \boldsymbol{a})=D+T \frac{a_{j}}{\kappa+\sum_{k \sim i} a_{k}}, & i \sim j, \\ q_{i j}(D, T, \boldsymbol{a})=0, & i \nsim j, i \neq j . \\ q_{i i}(D, T, \boldsymbol{a})=-\sum_{j \neq i} q_{i j}(D, T, \boldsymbol{a}), & \end{cases}
$$

We say that a matrix $\boldsymbol{A}=\left(a_{i, j}\right)_{i, j=1, \ldots, L}$ is irreducible if for any pair of nodes $(i, j)$, there is a path $i_{0}=i \rightarrow i_{1} \rightarrow i_{2} \rightarrow \ldots \rightarrow i_{k}=j$ such that $a_{i_{n} i_{n+1}}>0, n=$ $0, \ldots, k-1$. When $Q$ is irreducible, one can prove the existence of a unique invariant probability measure $\pi$ satisfying $\boldsymbol{\pi}^{*} \boldsymbol{Q}=0$, where ${ }^{*}$ denote a transposed vector (Norris 1997). In our context, a probability measure can be viewed as a probability vector i.e. a vector with non-negative entries that sum up to one.

Let us write (2) in the more compact form

$$
\frac{\mathrm{d} \boldsymbol{a}}{\mathrm{dt}}=f(\boldsymbol{a})=\boldsymbol{a}^{*} \boldsymbol{Q}(D, T, \boldsymbol{a}) .
$$

Hence, any solution of $f(\boldsymbol{a})=0$ is an invariant measure associated with the transition function $\boldsymbol{Q}(D, T, \boldsymbol{a})$. It can be seen that:

(R1) if $D>0$, the generator $\boldsymbol{Q}(D, T, \boldsymbol{a})$ is irreducible.

(R2) for pure transport processes $(D=0$ and $T>0), \boldsymbol{Q}(0, T, \boldsymbol{a})$ is irreducible if and only if $a_{i}>0 \forall i \in \Lambda$.

\subsection{The irreducible case}

In the irreducible case, let $\boldsymbol{\pi}(\boldsymbol{a})$ denote the associated positive invariant probability measure. The vector $\boldsymbol{a}>0$ is thus an equilibrium point of (2) if and only if

$$
\frac{a}{\sum_{i \in \Lambda} a_{i}}=\pi(a)
$$




\subsubsection{Pure transport processes}

An irreducible transition kernel $\boldsymbol{Q}$ of invariant probability measure $\pi$ is said to be reversible when

$$
\pi_{i} q_{i j}=\pi_{j} q_{j i}, \quad \forall i \neq j
$$

If $\boldsymbol{Q}(0, T, \boldsymbol{a})$ is reversible, the equation $f(\boldsymbol{a})=0$ is equivalent to the set of equations

$$
a_{i} q_{i j}(0, T, \boldsymbol{a})=a_{j} q_{j i}(0, T, \boldsymbol{a}), \quad i \neq j
$$

In the sequel, we shall use the notation

$$
N_{k}=N_{k}(\boldsymbol{a})=\kappa+\sum_{j \sim k} a_{j}
$$

Lemma 1 Let $G$ be a connected graph. Assume that $D=0$ and $T>0$.

Then $\boldsymbol{Q}(0, T, \boldsymbol{a})$ is reversible $\forall \boldsymbol{a}>0$, with invariant probability measure given by

$$
\boldsymbol{\pi}(\boldsymbol{a})=\left(\frac{a_{i} N_{i}}{Z(\boldsymbol{a})}\right)_{i \in \Lambda},
$$

where

$$
Z(\boldsymbol{a})=\sum_{i \in \Lambda} a_{i} N_{i}=\kappa \sum_{i \in \Lambda} a_{i}+\sum_{i \in \Lambda} \sum_{j \sim i} a_{i} a_{j}
$$

Moreover, $\boldsymbol{a}>0$ is an equilibrium point of (2) if and only if $N_{i}(\boldsymbol{a})$ does not depend on $i$, with

$$
N_{i}(\boldsymbol{a}) \equiv \frac{Z(\boldsymbol{a})}{\sum_{k \in \Lambda} a_{k}}=\kappa+\frac{\sum_{k \in \Lambda} \sum_{j \sim k} a_{k} a_{j}}{\sum_{k \in \Lambda} a_{k}}
$$

Remark 1 The transition rates $q_{i j}(\boldsymbol{a})$ are similar to the rates associated with a family of Markov chains used in the study of vertex-reinforced random walks, see Benaïm (1997), Benaïm and Tarrès (2008) and Pemantle (1992), and Lemma 1 is an adaptation of these results. Interestingly, such vertex-reinforced random walks are approximated by deterministic dynamical systems called replicator dynamics, of the form

$$
\frac{\mathrm{d} a_{i}}{\mathrm{~d} t}=a_{i}\left(N_{i}^{\prime}(\boldsymbol{a})-H^{\prime}(\boldsymbol{a})\right),
$$

where $N_{i}^{\prime}(\boldsymbol{a})=N_{i}(\boldsymbol{a})-\kappa$ and $H^{\prime}(\boldsymbol{a})=\sum_{i \in \Lambda} a_{i} N_{i}^{\prime}$. In this setting, the function $H^{\prime}$ plays the role of a Lyapunov function. We will also find a similar Lyapunov function, see Sect. 6. 
Proof Assume, without loss of generality, that $T=1$. First notice that

$$
\begin{aligned}
\sum_{j \sim i} \pi(\boldsymbol{a})_{j} q_{j i}(0, T, \boldsymbol{a}) & =\sum_{j \sim i} \frac{a_{j} N_{j}}{Z(\boldsymbol{a})} \frac{a_{i}}{N_{j}} \\
& =\frac{1}{Z(\boldsymbol{a})} \sum_{j \sim i} a_{i} a_{j}=\frac{a_{i}}{Z(\boldsymbol{a})} \sum_{j \sim i} a_{j}=\frac{a_{i}\left(N_{i}-\kappa\right)}{Z(\boldsymbol{a})} .
\end{aligned}
$$

The identity

$$
\pi(\boldsymbol{a})_{i} q_{i i}(0, T, \boldsymbol{a})=-\frac{a_{i} N_{i}}{Z(\boldsymbol{a})} \sum_{j \sim i} \frac{a_{j}}{N_{i}}=-\frac{a_{i}\left(N_{i}-\kappa\right)}{Z(\boldsymbol{a})},
$$

shows that

$$
\sum_{j \sim i} \pi(\boldsymbol{a})_{j} q_{j i}(0, T, \boldsymbol{a})+\pi(\boldsymbol{a})_{i} q_{i i}(0, T, \boldsymbol{a})=0
$$

so that $\boldsymbol{\pi}(\boldsymbol{a})$ is a invariant probability measure for $\boldsymbol{Q}(0, T, \boldsymbol{a})$. Moreover, $\boldsymbol{Q}(0, T, \boldsymbol{a})$ is reversible since

$$
\pi(\boldsymbol{a})_{i} q_{i j}(0, T, \boldsymbol{a})-\pi(\boldsymbol{a})_{j} q_{j i}(0, T, \boldsymbol{a})=\frac{a_{i} N_{i}}{Z(\boldsymbol{a})} \frac{a_{j}}{N_{i}}-\frac{a_{j} N_{j}}{Z(\boldsymbol{a})} \frac{a_{i}}{N_{j}}=0, \forall i \neq j .
$$

The point $\boldsymbol{a}>0$ is an equilibrium point (i.e. $f(\boldsymbol{a})=0$ ) if and only if $\frac{\boldsymbol{a}}{\sum_{i \in \Lambda} a_{i}}$ is an invariant measure for $\boldsymbol{Q}(0, T, \boldsymbol{a})$. Because of the uniqueness of the invariant measure, we obtain the required result $N_{i}(\boldsymbol{a}) \equiv \frac{Z(\boldsymbol{a})}{\sum_{k \in \Lambda} a_{k}}$.

Let $\boldsymbol{\Gamma}$ be the adjacency matrix of the graph $G=(\Lambda, E)$, that is, the matrix with entries given by $\gamma_{i j}=1$, when $i \neq j$ and $i \sim j$, and $\gamma_{i j}=0$ otherwise.

Lemma 1 states that $\boldsymbol{a}>0$ is an equilibrium point if and only if $N_{k}(\boldsymbol{a})$ does not depend on $k$, that is, see (7),

$$
\Gamma \boldsymbol{a}=\text { constant vector }=c(1, \ldots, 1)^{T},
$$

for some constant $c=c(\boldsymbol{a})$ since the constant may depend on $\boldsymbol{a}$. The following Corollary makes explicit this dependence:

Corollary 1 (Pure Transport Processes) Assume that $D=0$ and $T>0$ (no diffusion), and consider only positive $\boldsymbol{a}>0$. Then,

$$
f(\boldsymbol{a})=0, \quad \text { if and only if, } \quad \boldsymbol{\Gamma} \boldsymbol{a}=c(\boldsymbol{a}) \mathbf{1}, \quad \mathbf{1}=(1, \ldots, 1)^{*},
$$

where

$$
c(\boldsymbol{a})=\frac{\sum_{i \in \Lambda} \sum_{j \sim i} a_{i} a_{j}}{\sum_{i \in \Lambda} a_{i}}=\frac{\langle\boldsymbol{a}, \boldsymbol{\Gamma} \boldsymbol{a}\rangle}{\langle\boldsymbol{a}, \mathbf{1}\rangle} .
$$


Remark 2 Let $c$ be a constant, and let $\boldsymbol{a}$ (if it exists) be such that $\boldsymbol{\Gamma} \boldsymbol{a}=c \mathbf{1}$ and $\boldsymbol{a} \geq 0$. Then $\boldsymbol{a}$ is an equilibrium point and $c$ is given by (11).

Example 1 (The one-dimensional cycle) Assume that the $L$ cells are arranged on a cycle. The pure transport process $(D=0)$ is reversible for positive $\boldsymbol{a}>0$ and the equilibrium points $\boldsymbol{a}>0$ of system (2) are solutions of linear system (10). We give here some results developed in Sect. 7.1 to illustrate Corollary 1. When $L$ is a multiple of 4 , the set of equilibrium points $\boldsymbol{a} \in \mathbb{R}_{>0}^{L}$ having an auxin density $\rho>0$ (i.e. $\left.\rho=\sum_{i=1}^{L} a_{i} / L\right)$ forms a subset $M_{\rho}$ of $\mathbb{R}^{L}$ described with two parameters :

$$
\begin{gathered}
M_{\rho}=\left\{\left(a_{1}, a_{2},-a_{1}+2 \rho,-a_{2}+2 \rho, a_{1}, a_{2},-a_{1}+2 \rho,-a_{2}+2 \rho, \ldots\right) ;\right. \\
\left.a_{k} \in(0,2 \rho), k=1,2\right\} .
\end{gathered}
$$

When $L$ is not a multiple of $4, M_{\rho}$ reduces to the uniform configuration

$$
M_{\rho}=\{(\rho, \rho, \ldots, \rho)\}
$$

\subsubsection{General transport processes}

Lemma 2 Assume that $G$ is connected, and that both $D$ and $T$ are positive. For $\boldsymbol{a}>0$, $f(\boldsymbol{a})=0$ if and only if there exists a constant c such that $\boldsymbol{a}$ solves the following system of quadratic equations:

$$
\left(a_{i}-\frac{D}{T}\right) N_{i}(\boldsymbol{a})+a_{i}=c a_{i} N_{i}(\boldsymbol{a}), \quad i \in \Lambda .
$$

Proof Let $\mu_{i}=\left(a_{i}-D / T\right) N_{i}, i \in \Lambda$. Then $\boldsymbol{\mu}=\left(\mu_{i}\right)_{i \in \Lambda}$ satisfies

$$
\begin{aligned}
\left(\boldsymbol{\mu}^{*} \boldsymbol{Q}(0, T, \boldsymbol{a})\right)_{i} & =\sum_{j \sim i} \mu_{j} q_{j i}(\boldsymbol{a})+\mu_{i} q_{i i}(\boldsymbol{a}) \\
& =T \sum_{j \sim i}\left(a_{j}-\frac{D}{T}\right) N_{j} \frac{a_{i}}{N_{j}}-T\left(a_{i}-\frac{D}{T}\right) N_{i} \sum_{j \sim i} \frac{a_{j}}{N_{i}} \\
& =T \sum_{j \sim i}\left(a_{j}-\frac{D}{T}\right) a_{i}-T\left(a_{i}-\frac{D}{T}\right) \sum_{j \sim i} a_{j} \\
& =T \frac{D}{T} \sum_{j \sim i}\left(a_{j}-a_{i}\right)
\end{aligned}
$$

which gives the diffusive term contained in $f$. One can thus rewrite the equation $f(\boldsymbol{a})=0$ as

$$
(\boldsymbol{\mu}+\boldsymbol{a})^{*} \boldsymbol{Q}(0, T, \boldsymbol{a})=0 .
$$

By assumption, $\boldsymbol{a}>0$ so that $\boldsymbol{Q}(0, T, \boldsymbol{a})$ is irreducible as a Markov generator, and hence has only one invariant probability measure. The linear space composed of 
invariant measures is one-dimensional, so that the measure $\boldsymbol{\mu}+\boldsymbol{a}$ is proportional to $\boldsymbol{\pi}(\boldsymbol{a})$. The result is a consequence of (8).

\subsection{The reducible case}

We can adapt the previous notions to the case $D=0$ with reducible transition kernel $\boldsymbol{Q}(0, T, \boldsymbol{a})$, that is when at least one $a_{k}$ vanishes (see (R2)). We will see in the sequel that the $l$-stable equilibria are such that $a_{i}=0$ for some indices $i$ in $\Lambda$.

For arbitrary $i \in \Lambda$, the equilibrium points solve

$$
0=\sum_{k \sim i}\left(a_{k} \frac{a_{i}}{N_{k}(\boldsymbol{a})}-a_{i} \frac{a_{k}}{N_{i}(\boldsymbol{a})}\right) .
$$

If $a_{i}=0$, (13) is automatically satisfied, and if $a_{i} \neq 0$, (13) contains only terms associated with $k \sim i$ for which $a_{k} \neq 0$. Given $I \subset \Lambda$, we look for equilibria such that $a_{i}=0, i \in I$, and $a_{i}>0, i \in J=\Lambda \backslash I$. If one removes the nodes $i \in I$, the graph decomposes as a product of connected components $\gamma$, which form the subgraph of $G$ induced by the nodes of $J$. We will denote by $\left.\boldsymbol{a}\right|_{\gamma}$ the restriction of any $\boldsymbol{a}$ to the nodes $J_{\gamma}$ of $\gamma$. For each component $\gamma$, Corollary 1 shows that the related equilibrium points are obtained by solving linear systems of the form

$$
\left.\boldsymbol{\Gamma}_{\gamma} \boldsymbol{a}\right|_{\gamma}=\left.c_{\gamma} \mathbf{1}\right|_{\gamma}
$$

where $\boldsymbol{\Gamma}_{\gamma}$ is the adjacency matrix of the subgraph $\gamma$, and the $c_{\gamma}$ are normalization constants chosen in such a way that $\sum_{i} a_{i}=\rho L$, where $\rho$ is the density of auxin molecules. The set of equilibrium points is then obtained by taking the direct product of the sets of equilibrium points associated with the subgraphs $\gamma$.

\section{Stability of pure transport processes}

In this section, we study the stability of the equilibrium point obtained in the previous section for pure transport processes (i.e. $D=0$ ) on arbitrary graphs. Without loss of generality, we set $T=1$.

\subsection{The irreducible case}

We first discuss the stability of equilibrium points in the irreducible case. For such a point $\boldsymbol{a}$, we have $N_{i}(\boldsymbol{a}) \equiv N=\kappa+c$ and therefore, if the graph is regular, the uniform configuration $\boldsymbol{a}=\rho \mathbf{1}:=(\rho, \ldots, \rho)$ is an equilibrium point.

To get some idea on the stability, we consider the complete graph $K_{L}$ of $L$ nodes, and next turn to arbitrary graphs. In $K_{L}$, any pair of nodes $i \neq j$ are nearest neighbours; a simple computation shows that the Jacobian $\mathrm{d} f(\rho \mathbf{1})$ associated with (2) and evaluated at the uniform configuration $\rho \mathbf{1}$, is 


$$
\frac{\partial f_{i}(\rho \mathbf{1})}{\partial a_{j}}=\frac{\rho^{2}}{N^{2}}, \quad \frac{\partial f_{i}(\rho \mathbf{1})}{\partial a_{i}}=-\sum_{j \neq i} \frac{\rho^{2}}{N^{2}}, \quad \forall j \neq i .
$$

Consequently, $\mathrm{d} f(\rho \mathbf{1})$ is a symmetric matrix, and thus admits only non-positive real eigenvalues. The uniform configuration is therefore $l$-stable for the complete graph.

We next focus on arbitrary graphs. According to Corollary $1, \boldsymbol{a}>0$ is an equilibrium point if and only if $\Gamma \boldsymbol{a}=c(\boldsymbol{a}) \mathbf{1}$, for an explicit constant $c(\boldsymbol{a})$. Recall that $N=c(\boldsymbol{a})+\kappa$.

Proposition 3 Let $\boldsymbol{a}>0$ be an equilibrium point of (2) for $D=0$ and $T=1$. The Jacobian $\mathrm{d} f(\boldsymbol{a})=\left(\partial f_{i} / \partial a_{j}\right)$ evaluated at $\boldsymbol{a}$ is then given by

$$
d f(\boldsymbol{a})=\frac{1}{N^{2}} \operatorname{diag}(\boldsymbol{a}) \boldsymbol{\Gamma}(\operatorname{cid}-\operatorname{diag}(\boldsymbol{a}) \boldsymbol{\Gamma}),
$$

where diag $(\boldsymbol{a})$ is the diagonal matrix whose diagonal elements are given by $\boldsymbol{a}$, and where $\boldsymbol{\Gamma}$ is the adjacency matrix of the graph.

Proof See Appendix A.

Using the Jacobian matrix of (2) (Proposition 3) and the characterization of the spectral gap given in Diaconis and Stroock (1991), we derive a condition ensuring the non-stability of a configuration $\boldsymbol{a}$ satisfying $\boldsymbol{\Gamma} \boldsymbol{a}=c \mathbf{1}$ for some $c>0$.

Lemma 3 Let $G$ be a connected graph of adjacency matrix $\boldsymbol{\Gamma}$, and let $\boldsymbol{a}>0$ satisfy $\boldsymbol{\Gamma} \boldsymbol{a}=c \mathbf{1}$ for some $c>0$.

(a) The matrix $\boldsymbol{P}(\boldsymbol{a})$ defined by

$$
\boldsymbol{P}(\boldsymbol{a})=\frac{1}{c} \boldsymbol{\Gamma} \operatorname{diag}(\boldsymbol{a}),
$$

is stochastic (i.e. $\left.\sum_{j} P(\boldsymbol{a})_{i j}=1, \forall i\right)$, irreducible, reversible, with invariant measure $\boldsymbol{\pi}^{\prime}(\boldsymbol{a})$ given by $\boldsymbol{\pi}^{\prime}(\boldsymbol{a})_{i}=a_{i} /(\rho L)$, where $\rho=\left(\sum_{i=1}^{L} a_{i}\right) / L$, and with a real spectrum $-1 \leq \beta_{L} \leq \beta_{L-1} \leq \cdots \leq \beta_{2}<\beta_{1}=1$.

(b) Let $\mathcal{G}(\boldsymbol{a})$ be the spectral gap of $\boldsymbol{P}(\boldsymbol{a})$, defined by $\mathcal{G}(\boldsymbol{a})=1-\beta_{2}$.

Then $\boldsymbol{a}$ is a 1-stable configuration of (2) if and only if $\mathcal{G}(\boldsymbol{a}) \geq 1$.

(c) Moreover, the spectral gap is given by

$$
\mathcal{G}(\boldsymbol{a})=\delta \inf _{\phi} \frac{\sum_{i, j}(\phi(j)-\phi(i))^{2} \gamma_{i j} \pi^{\prime}(\boldsymbol{a})_{i} \pi^{\prime}(\boldsymbol{a})_{j}}{\sum_{i, j}(\phi(j)-\phi(i))^{2} \pi^{\prime}(\boldsymbol{a})_{i} \pi^{\prime}(\boldsymbol{a})_{j}},
$$

where $\delta=\rho L / c>1$, and where the infimum is taken over all nonconstant functions $\phi$.

Proof (a) The matrix $\boldsymbol{P}(\boldsymbol{a})$ is stochastic since by assumption $\boldsymbol{\Gamma} \boldsymbol{a}=c \mathbf{1}$. Then $\boldsymbol{P}(\boldsymbol{a})$ is reversible with invariant measure given by $\boldsymbol{\pi}^{\prime}(\boldsymbol{a})=\left(\frac{a_{i}}{\rho L}\right)_{i \in \Lambda}$. 
(b) The configuration $\boldsymbol{a}$ is $l$-stable if and only if the eigenvalues of the Jacobian matrix $\mathrm{d} f(\boldsymbol{a})$ given in Proposition 3 are all non-positive. The adjacency matrix $\boldsymbol{\Gamma}$ is symmetric, so that $(\boldsymbol{\Gamma} \operatorname{diag}(\boldsymbol{a}))^{*}=\operatorname{diag}(\boldsymbol{a}) \boldsymbol{\Gamma}$. It follows that the eigenvalues $\tilde{\beta}_{i}$ of $\operatorname{diag}(\boldsymbol{a}) \boldsymbol{\Gamma}$ are equal to $c \beta_{i}, i=1, \ldots, L$. The eigenvalues of $N^{2} \mathrm{~d} f(\boldsymbol{a})$ are given by $\tilde{\beta}_{i}\left(c-\tilde{\beta}_{i}\right)=\beta_{i}\left(1-\beta_{i}\right) c$. Hence, $\boldsymbol{a}$ is $l$-stable if and only if $\beta_{2}<0$, that is if and only if $\mathcal{G} \geq 1$.

(c) Notice next that

$$
A_{i j}=\pi^{\prime}(\boldsymbol{a})_{i} P(\boldsymbol{a})_{i j}=\delta \gamma_{i j} \pi^{\prime}(\boldsymbol{a})_{i} \pi^{\prime}(\boldsymbol{a})_{j},
$$

where $\delta=\rho L / c$ and we recall that $\gamma_{i j} \in\{0,1\}$ is the $(i, j)$ entry of the adjacency matrix $\boldsymbol{\Gamma}$.

Let $\mathcal{L}=$ id $-\boldsymbol{P}$ be the Laplace operator associated to $\boldsymbol{P}$, of eigenvalues $\lambda_{k}=$ $1-\beta_{k}, k=1, \ldots, L$. Then, the spectral gap $\mathcal{G}=1-\beta_{2}$ is given by (see Diaconis and Stroock 1991):

$$
\mathcal{G}=\lambda_{2}=\inf \left\{\frac{\mathcal{E}_{\pi^{\prime}}(\phi, \phi)}{\operatorname{Var}_{\pi^{\prime}}(\phi)}: \phi \text { is nonconstant }\right\}
$$

where

$$
\mathcal{E}_{\pi^{\prime}}(\phi, \phi)=\frac{1}{2} \sum_{i, j}(\phi(j)-\phi(i))^{2} A_{i j}
$$

is the Dirichlet form associated with $\mathcal{L}$, and where $\operatorname{Var}_{\pi^{\prime}}(\phi)$ is the variance of the random variable $\phi$ with respect to the invariant probability measure $\pi^{\prime}$. One can check that

$$
\operatorname{Var}_{\pi^{\prime}}(\phi)=\frac{1}{2} \sum_{i, j}(\phi(j)-\phi(i))^{2} \pi_{i}^{\prime} \pi_{j}^{\prime}
$$

We can also reformulate the above variational problem in a different way: set $\langle\phi\rangle_{\pi^{\prime}}=\sum_{i \in \Lambda} \phi(i) \pi_{i}^{\prime}$. Then

$$
\mathcal{G}=\inf \left\{\frac{\mathcal{E}_{\pi^{\prime}}(\phi, \phi)}{\operatorname{Var}_{\pi^{\prime}}(\phi)}:\langle\phi\rangle_{\pi^{\prime}}=0\right\}
$$

Hence, using this variational characterization of the spectral gap, we obtained that

$$
\mathcal{G} \leq \frac{\mathcal{E}_{\pi^{\prime}(\boldsymbol{a})}(\phi, \phi)}{\operatorname{Var}_{\pi^{\prime}(\boldsymbol{a})}(\phi)}=\delta \frac{\sum_{i, j}(\phi(j)-\phi(i))^{2} \gamma_{i j} \pi^{\prime}(\boldsymbol{a})_{i} \pi^{\prime}(\boldsymbol{a})_{j}}{\sum_{i, j}(\phi(j)-\phi(i))^{2} \pi^{\prime}(\boldsymbol{a})_{i} \pi^{\prime}(\boldsymbol{a})_{j}} \leq \delta,
$$

when $\phi$ is non-constant. 
Corollary 2 Let $G$ be a connected graph of adjacency matrix $\boldsymbol{\Gamma}$, and let $\boldsymbol{a}>0$ satisfy $\boldsymbol{\Gamma} \boldsymbol{a}=c \mathbf{1}$ for some $c>0$. For $i \in \Lambda$, let $\mathcal{N}_{i}=\{j \in \Lambda ; j \sim i\}$ be the neighbourhood of $i$. Assume that there exist elements $i_{0}, i_{1}, i_{2}$ and $i_{3}$ of $\Lambda$ such that

$$
i_{1} \in \mathcal{N}_{i_{0}}, \quad i_{2} \in\left(\mathcal{N}_{i_{1}} \backslash \mathcal{N}_{i_{0}}\right) \backslash\left\{i_{0}\right\}, i_{3} \in\left(\mathcal{N}_{i_{2}} \backslash \mathcal{N}_{i_{0}}\right) \backslash\left\{i_{0}\right\}
$$

\section{Then a is unstable.}

In other words, a configuration $\boldsymbol{a}>0$ is unstable if there is a path of length 3 $i_{0} \rightarrow i_{1} \rightarrow i_{2} \rightarrow i_{3}$ such that both $i_{2}$ and $i_{3}$ avoid $\left\{i_{0}\right\} \cup \mathcal{N}_{i_{0}}$. See Fig. $2 \mathrm{~b}$ for two examples of such paths.

Proof We use Lemma 3 to express the spectral gap of $\boldsymbol{P}(\boldsymbol{a})$ as

$$
\begin{aligned}
\mathcal{G}(\boldsymbol{a}) & =\delta \inf _{\langle\phi\rangle_{\pi^{\prime}(\boldsymbol{a})}=0} \frac{\sum_{i} \phi(i)^{2} \pi^{\prime}(\boldsymbol{a})_{i} \sum_{j} \gamma_{i j} \frac{a_{j}}{\rho L}-\sum_{i, j} \gamma_{i j} \phi(i) \phi(j) \pi^{\prime}(\boldsymbol{a})_{i} \pi^{\prime}(\boldsymbol{a})_{j}}{\sum_{i} \phi(i)^{2} \pi^{\prime}(\boldsymbol{a})_{i}} \\
& =\delta \inf _{\langle\phi\rangle_{\pi^{\prime}(\boldsymbol{a})}=0} \frac{\sum_{i} \phi(i)^{2} \pi^{\prime}(\boldsymbol{a})_{i} \frac{c}{\rho L}-\sum_{i, j} \gamma_{i j} \phi(i) \phi(j) \pi^{\prime}(\boldsymbol{a})_{i} \pi^{\prime}(\boldsymbol{a})_{j}}{\sum_{i} \phi(i)^{2} \pi^{\prime}(\boldsymbol{a})_{i}} \\
& =\delta \inf _{\langle\phi\rangle_{\pi^{\prime}(\boldsymbol{a})}=0} \frac{\sum_{i} \phi(i)^{2} \pi^{\prime}(\boldsymbol{a})_{i} \delta^{-1}-\sum_{i, j} \gamma_{i j} \phi(i) \phi(j) \pi^{\prime}(\boldsymbol{a})_{i} \pi^{\prime}(\boldsymbol{a})_{j}}{\sum_{i} \phi(i)^{2} \pi^{\prime}(\boldsymbol{a})_{i}}
\end{aligned}
$$

We will prove that $\mathcal{G}(\boldsymbol{a})<1$ by choosing a test function $\phi$ satisfying $\langle\phi\rangle_{\pi^{\prime}(\boldsymbol{a})}=0$ for which

$$
\delta \frac{\sum_{i} \phi(i)^{2} \pi^{\prime}(\boldsymbol{a})_{i} \delta^{-1}-\sum_{i, j} \gamma_{i j} \phi(i) \phi(j) \pi^{\prime}(\boldsymbol{a})_{i} \pi^{\prime}(\boldsymbol{a})_{j}}{\sum_{i} \phi(i)^{2} \pi^{\prime}(\boldsymbol{a})_{i}}<1,
$$

which is equivalent to

$$
\sum_{i, j} \gamma_{i j} \phi(i) \phi(j) \pi^{\prime}(\boldsymbol{a})_{i} \pi^{\prime}(\boldsymbol{a})_{j}>0
$$

We choose $i_{0} \in \Lambda$ such that (18) is satisfied. We set $\phi(j)=0, \forall j \in \mathcal{N}_{i_{0}}$. For all $k \in\left(\Lambda \backslash \mathcal{N}_{i_{0}}\right) \backslash\left\{i_{0}\right\}$, we choose $\phi(k)$ to be arbitrary but positive, in particular $\phi\left(i_{2}\right), \phi\left(i_{3}\right)>0$. Then we choose $\phi\left(i_{0}\right)$ so that

$$
a_{i_{0}} \phi\left(i_{0}\right)=-\sum_{j \neq i_{0}} a_{j} \phi(j)
$$

Consequently $\langle\phi\rangle_{\pi^{\prime}(\boldsymbol{a})}=0$ and $\sum_{i, j} \gamma_{i j} \phi(i) \phi(j) a_{i} a_{j}>0$.

Example 2 When $G$ is a subgraph of a two-dimensional grid of adjacency matrix $\Gamma$, a positive solution to the linear system $\boldsymbol{\Gamma} \boldsymbol{a}=c \mathbf{1}$ can only be $l$-stable when $G$ belongs to the list given in Fig. 2a, which consists in the square, the star, and all the various parts of the star. The reader can check this result by enumerating all the possible subgraphs and using Corollary 2. 

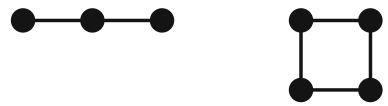

(a)
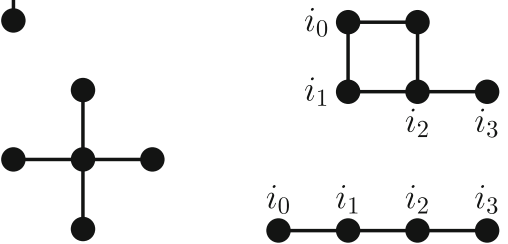

(b)

Fig. 2 a All possible subgraphs $\gamma$ of the two-dimensional grid that can potentially yield $l$-stable configurations. b Any configuration $\boldsymbol{a}>0$ will be unstable on these two subgraphs of the two-dimensional grid as a consequence of Corollary 2

\subsection{The reducible case}

We consider the reducible case where $a_{i}=0$ for $i \in I \subset \Lambda$. Set $J=\Lambda \backslash I$, and let $\left\{\gamma_{1}, \ldots, \gamma_{P}\right\}$ be the collection of subgraphs of $G$ induced by the nodes of $J$, of node set $J_{\gamma_{p}}$ and of adjacency matrices $\boldsymbol{\Gamma}_{\gamma_{p}}, p=1, \ldots, P$. We again assume that $\left.\boldsymbol{\Gamma}_{\gamma_{p}} \boldsymbol{a}\right|_{\gamma_{p}}=\left.c_{\gamma_{p}} \mathbf{1}\right|_{\gamma_{p}}$ for some $c_{\gamma_{p}}>0$.

The next proposition gives a stability criterion for the reducible case.

Proposition 4 Assume that $D=0$ and set $T=1$. Let $\boldsymbol{a}$ be an equilibrium point of (2) such that $a_{i}=0$ for $i \in I$. Let $\left\{\gamma_{1}, \ldots, \gamma_{P}\right\}$ be the collection of subgraphs of $G$ obtained by deleting the nodes of $I$, of adjacency matrices $\boldsymbol{\Gamma}_{\gamma_{p}}, p=1, \ldots, P$. The equilibrium points $\boldsymbol{a}$ are obtained by solving linear systems of the form $\left.\boldsymbol{\Gamma}_{\gamma_{p}} \boldsymbol{a}\right|_{\gamma_{p}}=$ $\left.c_{\gamma_{p}} \mathbf{1}\right|_{\gamma_{p}}$ for some $c_{\gamma_{p}}>0$ (see Sect. 4.2). The spectrum of the Jacobian evaluated at $\boldsymbol{a}$ is given by

$$
\operatorname{spec}(\mathrm{d} f(\boldsymbol{a}))=\bigcup_{p=1}^{P} \operatorname{spec}\left(\left.\mathrm{d} f\right|_{\gamma_{p}}\left(\left.\boldsymbol{a}\right|_{\gamma_{p}}\right)\right) \cup\left\{\sum_{k \sim i} \frac{a_{k}}{N_{k}}-\frac{N_{i}-\kappa}{N_{i}}, i \in I\right\}
$$

Proof See Appendix A.

Proposition 4 shows that such configurations are $l$-stable when

1) each $\left.\boldsymbol{a}\right|_{\gamma_{p}}$ is $l$-stable and

2) when $\sum_{k \sim i} a_{k} / N_{k}(\boldsymbol{a})-\left(N_{i}(\boldsymbol{a})-\kappa\right) / N_{i}(\boldsymbol{a}) \leq 0, i \in I$.

Here, if $k \sim i, i \in I, k \in \Lambda \backslash I, N_{k}(\boldsymbol{a})$ is given by the constant $\kappa+c_{\gamma_{p}}$ when $k \in J_{\gamma_{p}}$. To go further, we need the following

Definition 2 Let $J \subset \Lambda$. The outer boundary of $J$, denoted by $\partial J$, is the subset of $\Lambda$ given by

$$
\text { д } J=\{j \in \Lambda \backslash J \mid j \sim J\} .
$$




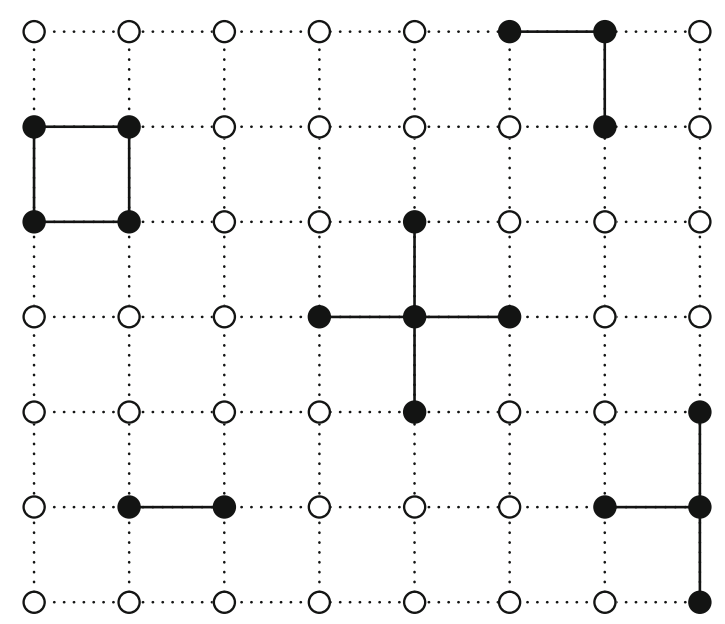

Fig. 3 A potentially $l$-stable configuration when the graph $G$ is a rectangular grid, for the pure transport process. The white circles correspond to the values $a_{i}=0, i \in I$ (the set of auxin depleted cells), while the black circles are such that $a_{i}>0$, corresponding to auxin peaks. One can construct the set of all $l$-stable configurations by playing with the building block given by the square, the star, and the various parts of the star. This shows that system (2) does not necessarily produce regular patterns. We can however, give examples where such configurations are unstable, see Sect. 5.3

\subsection{Example: The rectangular grid}

We now illustrate the various $l$-stable patches we can form by using the building blocks, as given in Figs. 2 and 3. It is easy to provide examples of potentially unstable configurations when the outer boundary of some component $\gamma$ is such that

$$
\partial\left(\partial J_{\gamma}\right) \cap J_{\gamma^{\prime}} \neq \emptyset, \quad \text { for some component } \quad \gamma^{\prime} \neq \gamma,
$$

as illustrated in Fig. 4a.

Next, the reader can verify, using Proposition 4, that any patch composed of building blocks disposed in such a way that

$$
\partial\left(\partial J_{p} \cup J_{p}\right) \cap\left(\cup_{p^{\prime} \neq p} J_{p^{\prime}}\right)=\emptyset, \quad \forall p=1, \ldots, P,
$$

is $l$-stable, if each blocks are also $l$-stable. Figure $4 \mathrm{~b}$ exhibits a typical example of a $l$-stable configuration in this setting.

Remark 3 The set of auxin depleted cells isolate the patches, so it is easy to see that a $l$-stable configuration on an cylinder is of the same type as a potentially $l$-stable configuration on a rectangular domain.

\section{Asymptotic properties of the auxin flux}

In this section, we investigate the behaviour of solutions of (2) on a graph when $t \rightarrow \infty$. The first result concerns the general process (with diffusion). 


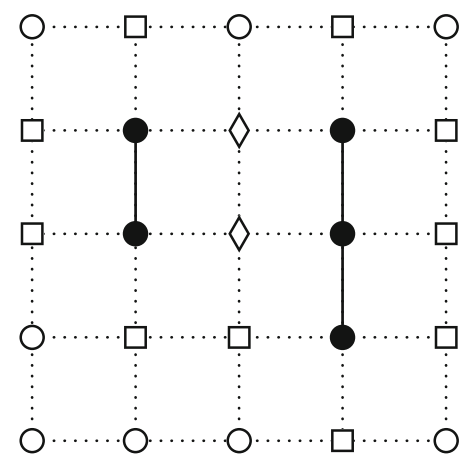

(a) Potentially unstable configuration

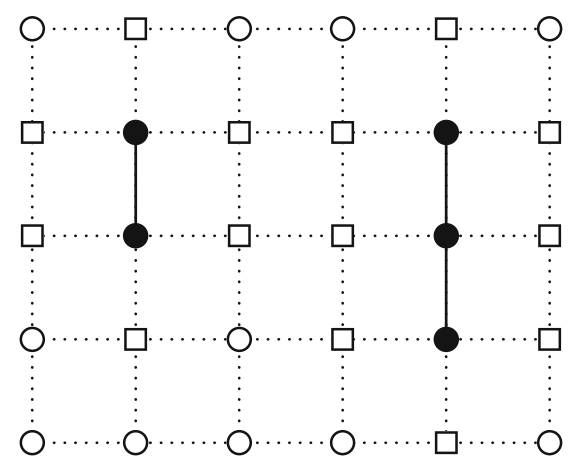

(b) Potentially $l$-stable configuration

Fig. 4 Black circles indicate cells with $a_{i}>0$ while white circles, squares and diamonds denote empty cells. Squares indicate neighbourhood of one non-zero patch and diamonds common neighbourhood of two non-zero patches. a One can check in this example that (20) implies the non-stability of the configuration for well chosen parameters. b In that case, (21) is satisfied, ensuring the stability of the configuration if each patch composed of black circles is $l$-stable

Proposition 5 Let us assume that the graph is connected and $D>0$. If $\sum_{k=1}^{L} a_{k}(0)>0$, then for all $i \in\{1, \ldots, L\}$, we have $\lim _{i n f} \operatorname{int}_{t \rightarrow \infty} a_{i}(t)>0$.

Proof See Appendix A.

As a consequence of Proposition 5, for $D>0$, it is impossible to have $\lim _{t \rightarrow+\infty} a_{i}(t)=0$, and thus none of the compartments can become empty asymptotically.

\subsection{The pure transport process}

We now prove the convergence of the solutions of system (2) to the set of equilibrium points for the pure transport process. Without loss of generality, suppose that $T=1$.

Theorem 1 (a) Let $\boldsymbol{a}(t)$ be the unique solution of (2) with $\boldsymbol{a}(0) \in \Omega$. Then $\boldsymbol{a}(t) \in \Omega$, $\forall t>0$ and $\boldsymbol{a}(t)$ converges to set of equilibrium points as $t \rightarrow \infty$.

(b) Moreover, every limit point of a trajectory $\boldsymbol{a}(t)$ is an equilibrium point i.e. if for $t_{n} \nearrow \infty, \boldsymbol{a}\left(t_{n}\right) \rightarrow \boldsymbol{a}_{\infty}$ then $\boldsymbol{a}_{\infty}$ is an equilibrium point.

To prove the Theorem 1, we use the method of Lyapunov functions.

Definition 3 A $C^{1}$-function $H(\boldsymbol{x})$ is a Lyapunov function for the o.d.e. $\frac{\mathrm{d} \boldsymbol{x}(t)}{\mathrm{d} t}=f(\boldsymbol{x})$ if

$$
\frac{\mathrm{d} H(\boldsymbol{x}(t))}{\mathrm{d} t}=\left\langle\nabla H(\boldsymbol{x}(t)), \frac{\mathrm{d} \boldsymbol{x}(t)}{\mathrm{d} t}\right\rangle \leq 0, \quad \forall t>0,
$$

for every solution of the o.d.e. in the domain of interest. 
Lemma 4 Assume that $D=0$ and set $T=1$. The function $H$ defined by

$$
H(\boldsymbol{a})=-\frac{1}{2} \sum_{k \in \Lambda} a_{k}\left(N_{k}(\boldsymbol{a})+\kappa\right)=-\kappa \sum_{k \in \Lambda} a_{k}-\frac{1}{2} \sum_{k} \sum_{j \sim k} a_{j} a_{k},
$$

is a Lyapunov function for system (2).

Proof Notice that

$$
\frac{\partial H}{\partial a_{k}}(\boldsymbol{a})=-N_{k}(\boldsymbol{a})
$$

because the function $N_{k}=N_{k}(\boldsymbol{a})=\kappa+\sum_{j \sim k} a_{j}$ does not depend on the variable $a_{k}$.

$$
\begin{aligned}
\frac{\mathrm{d} H(\boldsymbol{a}(t))}{\mathrm{d} t} & =-\sum_{k \in \Lambda} N_{k} \sum_{j \sim k}\left(a_{j} \frac{a_{k}}{N_{j}}-a_{k} \frac{a_{j}}{N_{k}}\right)=-\sum_{k \in \Lambda} N_{k} \sum_{j \sim k} \frac{a_{j}}{N_{k}} \frac{a_{k}}{N_{j}}\left(N_{k}-N_{j}\right) \\
& =-\sum_{k \in \Lambda} N_{k} \sum_{j \sim k} \frac{a_{j}}{N_{k}} \frac{a_{k}}{N_{j}}\left(N_{k}-N_{j}\right) \\
& =-\frac{1}{2} \sum_{k \in \Lambda} \sum_{j \sim k} \frac{a_{j}}{N_{k}} \frac{a_{k}}{N_{j}}\left(N_{k}\left(N_{k}-N_{j}\right)+N_{j}\left(N_{j}-N_{k}\right)\right) \\
& =-\frac{1}{2} \sum_{k \in \Lambda} \sum_{j \sim k} \frac{a_{j}}{N_{k}} \frac{a_{k}}{N_{j}}\left(N_{k}-N_{j}\right)^{2} .
\end{aligned}
$$

By Proposition $1, a_{i}(0) \geq 0, \forall i$, implies that $a_{i}(t) \geq 0, \forall i, \forall t>0$, proving the assertion.

The asymptotic behaviour of the auxin flux will be derived from the theorem of LaSalle (1960). Introduce the notation

$$
\dot{H}(\boldsymbol{a})=\sum_{i=1}^{L} \frac{\partial H}{\partial a_{i}} f_{i}(\boldsymbol{a})=-\frac{1}{2} \sum_{k \in \Lambda} \sum_{j \sim k} \frac{a_{j}}{N_{k}} \frac{a_{k}}{N_{j}}\left(N_{k}-N_{j}\right)^{2} .
$$

For an arbitrary $\rho>0$, consider the sets

$$
\Omega=\left\{\boldsymbol{x} \in[0, \rho L]^{L} \mid \sum_{i} x_{i}=\rho L\right\} \quad \text { and } E_{\Omega}=\{\boldsymbol{x} \in \Omega \mid \dot{H}(\boldsymbol{x})=0\} .
$$

Lemma 5 The set $E_{\Omega}$ is the set of equilibrium points for system (2).

Proof Let $\boldsymbol{a} \in \Omega$. Then $\dot{H}(\boldsymbol{a})=0$ if and only if for all pairs $j \sim k$, either $a_{j}=0$, $a_{k}=0$ or $N_{j}=N_{k}$. Let $I_{a}:=\left\{i \in \Lambda ; a_{i}=0\right\}$. Then $\dot{H}(\boldsymbol{a})=0$ if and only if, for all pairs of neighbours $j \sim k$ such that $j \in \Lambda \backslash I_{a}$ and $k \in \Lambda \backslash I_{a}$, one has $N_{j}=N_{k}$. Let $\gamma$ be the connected component of the graph containing this pair (see Sect. 4.2), with 
$N_{j}=N_{k}=c_{\gamma}$, for some positive constant $c_{\gamma}$. Then, $N_{i} \equiv c_{\gamma}, \forall i \in \gamma$. Consequently $\dot{H}(\boldsymbol{a})=0$ if and only if the function $N$ is constant on the connected components $\gamma$ associated with $I_{a}$. Hence, for each such component, $\left.\boldsymbol{\Gamma}_{\gamma} \boldsymbol{a}\right|_{\gamma}=\left.c_{\gamma} \mathbf{1}\right|_{\gamma}$. The results is a consequence of Corollary 1 and of the results of Sect. 4.2.

Let $M_{\Omega}$ be the largest invariant subset of $E_{\Omega}$ i.e. every solution of (2) starting with $\boldsymbol{a}(0) \in M_{\Omega}$ stays in $M_{\Omega}$ for all future time. As $E_{\Omega}$ contains only the equilibrium points of $f, E_{\Omega}$ is invariant. Hence, $M_{\Omega}=E_{\Omega}$.

We recall the invariance theorem of LaSalle (1960).

Theorem 2 (LaSalle) Let $\Omega$ be a compact set with the property that every solution of (2) which begins in $\Omega$ remains for all future time in $\Omega$. Let $H: \Omega \rightarrow \mathbb{R}$ be a Lyapunov function on $\Omega$ for (2). Then every solution starting in $\Omega$ converges to $M_{\Omega}$ as $t \rightarrow \infty$.

We are now ready to prove the Theorem 1 .

Proof Proposition 1 shows that the compact set $\Omega$ is invariant. The continuously differentiable function $H$ is a Lyapunov function by the Lemma 5.Then (a) follows directly from Theorem 2 .

If $\boldsymbol{a}_{\infty} \notin \Omega$, as $E_{\Omega}=M_{\Omega}$ is a closed set then $\operatorname{dist}\left(\boldsymbol{a}_{\infty}, E_{\Omega}\right)>0$, a contradiction with (a) which proves (b).

Remark 4 (Global minimizers of $H$ ) The literature contains results on the set $\mu(G)$ of minimizers of $H$ when $\sum_{i \in \Lambda} a_{i}=1$. The authors of Motzkin and Straus (1965) proved that $\max _{\boldsymbol{a}}\langle\boldsymbol{a}, \boldsymbol{\Gamma} \boldsymbol{a}\rangle=(\omega(G)-1) / \omega(G)$, where $\omega(G)$ is the clique number of $G$, that is the order of the largest complete subgraph of $G$. They also proved that the absolute minimum of $H$ is achieved at an interior point of the unit simplex if and only if $G$ is a complete multipartite graph. Various results were obtained in Waller (1977). For example, $\mu(G)$ is a simplicial complex having an automorphism group similar to that of $G$. In some sense, $\mu(G)$ mirrors some of the geometry of the graph $G$.

Proposition 6 System (2) does not admit non-constant periodic solutions if $D=0$.

Proof Every point of a periodic solution is a limit point and, according to Theorem 1, it is an equilibrium point. Uniqueness of a solution provides a contradiction.

Proposition 7 For a fixed auxin density $\rho>0$, the set of equilibrium points of (2), $E_{\Omega}$, is uncountable if $D=0$ and the graph $G$ is not complete.

Proof Let $i_{1}, i_{2}$ two different non-neighbouring cells. For a fixed $\rho>0$, the set $E_{\Omega}$ is uncountable as it contains the following configurations:

$$
\left\{a \in \Omega \mid a_{i_{1}}=c_{1}, a_{i_{2}}=\rho L-c_{1} \text { with } c_{1} \in(0, \rho L)\right\}
$$

Remark 5 If the graph is complete then the matrix $\Gamma=\mathbf{1}-\boldsymbol{i} d$ is invertible for $L>1$ and the equation $\Gamma \boldsymbol{a}=c \mathbf{1}$ has an unique solution, the uniform one. 


\section{The process on the circle}

We study in more details the process on the circle. This can be motivated by the fact that the patterning process of phyllotaxis takes place on the epidermis of the meristem which is approximately an annulus of cells (Reinhardt et al. 2000).

7.1 The pure transport process on the circle

Let $D=0$ and $T=1$. Corollary 2 yields the instability of the uniform solution $\rho \mathbf{1}=(\rho, \ldots, \rho)$ when the length $L$ of the cycle is larger than 4 . In the following Corollary, we give the other equilibrium points of (2) and conclude about their stability in the irreducible case.

Corollary 3 Assume that the nodes are arranged on a circle of size L. The set $M_{\rho}$ of equilibrium points $\boldsymbol{a}>0$ such that $f(\boldsymbol{a})=0$ and $\sum_{i} a_{i}=\rho L$ contains only the uniform configuration $(\rho, \ldots, \rho)$ if $L$ is not a multiple of 4 . If $L=4 n$, for some $n \in \mathbb{N}$ with $n \geq 1, M_{\rho}$ is given by

$$
\begin{aligned}
M_{\rho}=\left\{\left(a_{1}, a_{2},-a_{1}+2 \rho,-a_{2}+2 \rho, a_{1}, a_{2},-a_{1}+2 \rho,\right.\right. & \left.-a_{2}+2 \rho, \ldots\right) ; \\
& \left.a_{k} \in(0,2 \rho), k=1,2\right\} .
\end{aligned}
$$

Any element of $M_{\rho}$ is unstable except when $L=4$.

Proof The adjacency matrix of the circle is circulant, with eigenvalues given by

$$
\mu_{k}=e^{2 \pi i \frac{k}{L}}+e^{2 \pi i \frac{(L-1) k}{L}}=2 \cos \left(2 \pi \frac{k}{L}\right) .
$$

The determinant of $\boldsymbol{\Gamma}$ vanishes if and only if there exists $j \in\{1, \ldots, L\}$ such that $\mu_{j}=0$, that is if $L$ is a multiple of 4 .

Let

$$
M_{\rho}=\left\{\boldsymbol{a} \in \mathbb{R}^{L} \text { equilibrium point of (2); } a_{i}>0, \forall i \in \Lambda, \sum_{i=1}^{L} a_{i}=\rho L\right\}
$$

For every $\boldsymbol{a} \in M_{\rho}$, we have

$\boldsymbol{a}=\left(a_{1}, a_{2}, 2 \rho-a_{1}, 2 \rho-a_{2}, a_{1}, a_{2}, 2 \rho-a_{1}, 2 \rho-a_{2}, \ldots, a_{1}, a_{2}, 2 \rho-a_{1}, 2 \rho-a_{2}\right)$

with $\left(a_{1}, a_{2}\right) \in(0,2 \rho) \times(0,2 \rho)$. Corollary 2 implies that this set contains only unstable points when $L>4$.

For $L=4$, the equilibrium point $\boldsymbol{a}=\left(a_{1}, a_{2}, 2 \rho-a_{1}, 2 \rho-a_{2}\right)$ is $l$-stable since the eigenvalues of the Jacobian matrix are 


$$
\lambda_{1}=\lambda_{2}=\lambda_{3}=0 \text { and } \lambda_{4}=-\frac{2 c}{(\kappa+c)^{2}} .
$$

The set $M_{\rho}^{\text {tot }}$ of all equilibrium points is obtained by decomposing the circle into subgraph $\gamma$ such $\left.\boldsymbol{a}\right|_{\gamma}>0$ and by solving the system

$$
\left.\boldsymbol{\Gamma}_{\gamma} \boldsymbol{a}\right|_{\gamma}=\left.c_{\gamma} \mathbf{1}\right|_{\gamma}
$$

for these subgraphs. We can prove that this system has positive solution $\left.\boldsymbol{a}\right|_{\gamma}$ if and only $\left|J_{\gamma}\right|<4$, where $\left|J_{\gamma}\right|$ is the number of nodes associated to the subgraph $\gamma$. Indeed for $\left|J_{\gamma}\right| \geq 4$, we can see that $a_{4}$ must be 0 , which is in contradiction with the hypothesis. When $\left|J_{\gamma}\right|=3$, the equilibrium points take the form $\left.\boldsymbol{a}\right|_{\gamma}=\left(z_{1}, c_{\gamma}, c_{\gamma}-z_{1}\right)$, with $z_{1} \in\left(0, c_{\gamma}\right)$ and when $\left|J_{\gamma}\right|=2,\left.\boldsymbol{a}\right|_{\gamma}=\left(c_{\gamma}, c_{\gamma}\right)$. In these two cases, the equilibrium points are $l$-stable as the Lyapunov function $H$ defined in (22) takes its minimal value $H(\boldsymbol{a})=-\left(\kappa+\frac{\rho L}{4}\right) \rho L$. The global minimum of $H$ is obtained by adapting the result of Motzkin and Straus (1965), see Remark 4. Finally, if $\left|J_{\gamma}\right|=1$, we have $\left.\boldsymbol{a}\right|_{\gamma}=\left(c_{\gamma}\right)$; $H$ is maximal but not strictly.

The set $M_{\rho}^{\text {tot }}$ of equilibrium points is then obtained by taking the direct product of the sets of equilibrium configurations associated with the paths $\gamma$. For example, if $L$ is a multiple of 4 , the subset of $M_{\rho}^{\text {tot }}$ defined by

$$
\begin{array}{r}
\tilde{M}_{\rho}=\left\{\left(a_{1}, a_{2},-a_{1}+2 \rho,-a_{2}+2 \rho, a_{1}, a_{2},-a_{1}+2 \rho,-a_{2}+2 \rho, \ldots\right) ;\right. \\
\left.a_{1}=0, a_{2} \in(0,2 \rho)\right\},
\end{array}
$$

is composed of equilibrium points which are $l$-stable since

$$
\lambda=0 \text { with multiplicity } 3 \frac{L}{4} \text { and } \lambda=\frac{-2 c^{2}}{(\kappa+c)^{2}} \text { with multiplicity } \frac{L}{4}
$$

\subsection{Explicit computations on the circle when $D=0$}

As we have seen, when the number of nodes $J_{\gamma}$ associated to the subgraph $\gamma$ is $\left|J_{\gamma}\right|=3$, the $l$-stable configurations are given by triplets of the form $\left(z_{1}, c_{\gamma}, c_{\gamma}-z_{1}\right)$, where $z_{1}$ is such that $z_{1} \in\left[0, c_{\gamma}\right]$, for some positive constant $c_{\gamma}>0$.

Consider a path composed of five cells $i_{0}-1, i_{0}, i_{0}+1, i_{0}+2$ and $i_{0}+3$ such that $a_{i_{0}-1}=a_{i_{0}+3}=0$. Every solution of (2) starting with such a configuration converges on $\gamma$ to an equilibrium point.

Proposition 8 We consider system (2) on the circle with $D=0$, starting with an initial configuration such $a_{i_{0}-1}(0)=a_{i_{0}+3}(0)=0, a_{i_{0}}(0), a_{i_{0}+1}(0)>0, a_{i_{0}+2} \geq 0$ and $\rho=$ $\frac{1}{3} \sum_{k=i_{0}}^{i_{0}+2} a_{k}(0)$. Let $\gamma$ be the subgraph associated to the nodes $J_{\gamma}:=\left\{i_{0}, i_{0}+1, i_{0}+2\right\}$, then the orbit $\left.\boldsymbol{a}\right|_{\gamma}(t)$ converges to

$$
\left(z_{1}, c_{\gamma}, c_{\gamma}-z_{1}\right), \text { with } z_{1}=\frac{c_{\gamma}}{1+c}, c_{\gamma}=\frac{3 \rho}{2} \text { and } c=\frac{a_{i_{0}+2}(0)}{a_{i_{0}}(0)} .
$$


Notice that if $a_{i_{0}+1}(0)=0$, then the orbit $\left.\boldsymbol{a}\right|_{\gamma}(t)$ stays at $\left.\boldsymbol{a}\right|_{\gamma}(0)$.

Proof The system (2) associated with the cells $\left\{i_{0}, i_{0}+1, i_{0}+2\right\}$ becomes

$$
\begin{aligned}
\frac{\mathrm{d} a_{i_{0}}}{\mathrm{~d} t} & =a_{i_{0}}\left(\frac{a_{i_{0}+1}}{\kappa+a_{i_{0}}+a_{i_{0}+2}}-\frac{a_{i_{0}+1}}{\kappa+a_{i_{0}+1}}\right), \\
\frac{\mathrm{d} a_{i_{0}+2}}{\mathrm{~d} t} & =a_{i_{0}+2}\left(\frac{a_{i_{0}+1}}{\kappa+a_{i_{0}}+a_{i_{0}+2}}-\frac{a_{i_{0}+1}}{\kappa+a_{i_{0}+1}}\right), \\
\frac{\mathrm{d} a_{i_{0}+1}}{\mathrm{~d} t} & =\frac{a_{i_{0}} a_{i_{0}+1}}{\kappa+a_{i_{0}+1}}+\frac{a_{i_{0}+2} a_{i_{0}+1}}{\kappa+a_{i_{0}+1}}-\frac{a_{i_{0}+1} a_{i_{0}}}{\kappa+a_{i_{0}}+a_{i_{0}+2}}-\frac{a_{i_{0}+1} a_{i_{0}+2}}{\kappa+a_{i_{0}}+a_{i_{0}+2}} .
\end{aligned}
$$

These three equations entail the existence of a constant $c>0$ such that

$$
a_{i_{0}+2}=c a_{i_{0}}
$$

and

$$
a_{i_{0}+1}=3 \rho-(1+c) a_{i_{0}}
$$

Setting $u(t)=(1+c) a_{i_{0}}(t)$, one gets

$$
\frac{\mathrm{d} u}{\mathrm{~d} t}=\frac{u(3 \rho-u)(3 \rho-2 u)}{(\kappa+u)(3 \rho+\kappa-u)} \quad u(0)>0 .
$$

Since $a_{i_{0}}(t), a_{i_{0}+1}(t)>0$ for all $t \geq 0$, we deduce that $0<u(t)<3 \rho$

Lemma 6 As $t \rightarrow \infty, u(t)=(1+c) a_{i_{0}}(t) \longrightarrow \frac{3 \rho}{2}$.

Proof Clearly 0, $\frac{3 \rho}{2}$ and $3 \rho$ are the equilibrium points of Eq. (28). Let

$$
g(u)=\frac{u(3 \rho-u)(3 \rho-2 u)}{(\kappa+u)(3 \rho+\kappa-u)}
$$

with $u \in(0,3 \rho)$.

We can easily find a compact interval $I_{0}$ whose interior contains $(0,3 \rho)$ such that $g^{\prime}$ is continuous and bounded over $I_{0}$. As a consequence $g$ satisfies a Lipschitzcondition over $I_{0}$. According to the general theory of o.d.e.'s, for any initial condition $u(0) \in(0,3 \rho) \mathrm{Eq}$. (28) admits a unique solution defined over a maximal interval $I_{m}$. If $u(0) \in\left(0, \frac{3 \rho}{2}\right)$, then $\dot{u}(0)>0$. Due to uniqueness, the solution cannot reach an equilibrium point in a finite time and thus the boundary of $\left(0, \frac{3 \rho}{2}\right)$. Moreover the solution is obviously bounded entailing $I_{m}=[0,+\infty[$. For the preceding reasons the derivative of $u(t)$ is never 0 and thus always positive since $\dot{u}(0)>0$. Thus $u(t)$ increases to $\frac{3 \rho}{2}$ as $t \rightarrow+\infty$. The same reasoning shows that $u(t)$ decreases to $\frac{3 \rho}{2}$ as $t \rightarrow+\infty$ for $u(0) \in\left(\frac{3 \rho}{2}, 3 \rho\right)$. 
Furthermore, (26) yields

$$
c=\frac{a_{i_{0}+2}(0)}{a_{i_{0}}(0)} .
$$

As $(1+c) a_{i_{0}}(t)=a_{i_{0}}(t)+a_{i_{0}+2}(t)$ tends to $c_{\gamma}$ as $t \rightarrow \infty$, Lemma 6 yields $c_{\gamma}=3 \rho / 2$, and

$$
a_{i_{0}}(t) \underset{t \rightarrow \infty}{\longrightarrow} \frac{3 \rho}{2(1+c)}=\frac{c_{\gamma}}{1+c}
$$

(26) and (27) entail

$$
\begin{aligned}
& a_{i_{0}+1}=3 \rho-(1+c) a_{i_{0}} \underset{t \rightarrow \infty}{\longrightarrow} \frac{3 \rho}{2}=c_{\gamma} \\
& a_{i_{0}+2}=c a_{i_{0}} \underset{t \rightarrow \infty}{\longrightarrow} \frac{c}{1+c} c_{\gamma}=c_{\gamma}-\frac{c_{\gamma}}{1+c} .
\end{aligned}
$$

In summary, every orbit with an initial condition of the form

$$
\left(a_{i_{0}-1}(0), a_{i_{0}}(0), a_{i_{0}+1}(0), a_{i_{0}+2}(0), a_{i_{0}+3}(0)\right)
$$

with $a_{i_{0}-1}(0)=a_{i_{0}+3}(0)=0$ and $a_{i_{0}}(0), a_{i_{0}+1}(0)>0$, converges to the equilibrium point $\left(z_{1}, c_{\gamma}, c_{\gamma}-z_{1}\right)$, with $z_{1}=\frac{c_{\gamma}}{1+c}, c_{\gamma}=\frac{3 \rho}{2}$ and $c=\frac{a_{i_{0}+2}(0)}{a_{i_{0}}(0)}$.

\section{Possible extension: inclusion of potentials}

As stated in the Introduction, experiments have shown that both mechanical and biochemical processes play a role in plant patterning. We here adapt some ideas of Newell et al. (2008) and Newell and Shipman (2005) to our discrete setting. The former considered the discrete model (2) and by taking a continuous limit, they obtain a p.d.e. describing the time evolution of auxin concentrations. The latter is linked to the von Karman equations from elasticity theory. These equations describe the deformations of an elastic shell or plate subject to various loading conditions. Usually, the in-plane stress is described with Airy functions which are potential for the stress field. Here, we will simply suppose that this potential is given by some function $\left(\phi_{i}\right)_{1 \leq i \leq L}$. We also suppose that the auxin flux is directed in part by these potentials and assume a model of the form

$$
\frac{\mathrm{d} a_{i}(t)}{\mathrm{d} t}=f_{i}(\boldsymbol{a})+\sum_{j \sim i}\left(a_{j} \phi_{i}-a_{i} \phi_{j}\right),
$$

$i=1, \ldots, L$. We have seen that the equilibrium points of (2) exhibits locally regular geometrical patterns, but not necessarily globally. The potential might be chosen to reproduce the patterns obtained when considering mechanical buckling, and model (29) might then lead to more regularly spaced auxin peaks, see Fig. 5. 


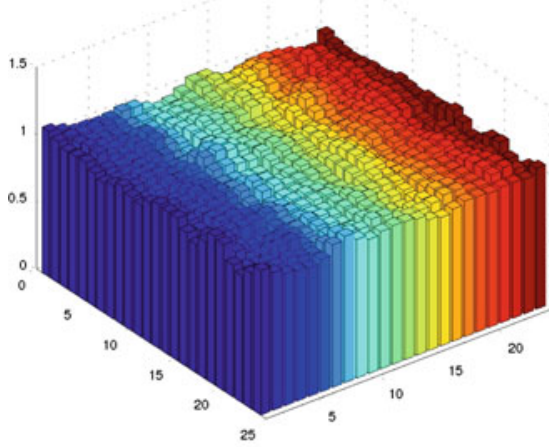

(a) $a(t)$ for $t \approx 0$

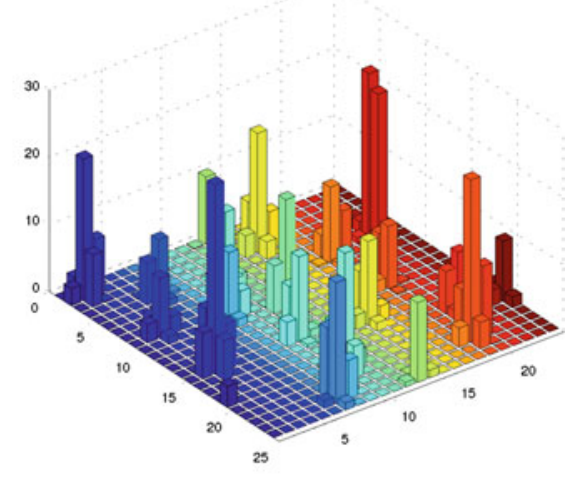

(b) $\boldsymbol{a}(t)$ for large $t$.

Fig. 5 Simulation of the orbits of the differential equation (29) with $T=1, D=0$ and a potential $\phi(x, y)=\sin (4 \pi x / A) \sin (4 \pi y / B)$ on a torus, where $x=1, \ldots, A$ and $y=1, \ldots, B$. The initial state is flat. $\mathbf{b}$ shows the state $\boldsymbol{a}(t)$ for large $t$ : one sees regularly spaced auxin peaks, which are isolated in a background of auxin depleted cells. The potential and transport terms drift thus the process toward more regular patterns, while the transport process creates domains of auxin depletion

Lemma 7 Assume a model of the form (29), with $D>0$ and $T>0$. Let $\boldsymbol{a}>0$. Then $f_{i}(\boldsymbol{a})+\sum_{j \sim i}\left(a_{j} \phi_{i}-a_{i} \phi_{j}\right)=0$ if and only if there exists a constant $c \in \mathbb{R}$ such that

$$
\left(a_{i}-\frac{D}{T}-\frac{1}{T} \phi_{i}\right) N_{i}(\boldsymbol{a})+a_{i}=c a_{i} N_{i}(\boldsymbol{a}), \quad i \in \Lambda .
$$

Proof The proof of Lemma 7 is identical with the proof of Lemma 2.

\section{Conclusion}

Auxin is thought to be one of the main actors of plant patterning, but is almost undetectable at present time. This is thus a particularly interesting field where mathematical models might be instrumental both for understanding the output of biological experiments and for developing new biological scenarios. One leading idea is that the auxin flux is directed by active transport, where PIN proteins help guiding auxin molecules to create auxin accumulation points where new primordia emerge. As explained in the Introduction, patterns in plant are highly regular geometrically, and the problem of understanding these arrangements is still challenging. We focused on a leading model of auxin transport as given by the o.d.e. (2), and studied the associated $l$ stable equilibria of this dynamical system. In this model, the action of PIN proteins is only considered through an adiabatic limit. We proved that the $l$-stable equilibria are composed of patches of locally regular patterns, which are isolated in a sea of auxin depleted cells, see e.g. Fig. 4a. It transpires that these patterns are not in general geometrically regular. The more involved mathematical model of Smith et al. (2006) also considers active auxin transport by incorporating PIN proteins, which are 
dynamically redistributed along cell membranes. In this last model, the distribution of PIN proteins is defined using exponential weights, and simulations indicate that the obtained patterns are more regular. This is a strong assumption, which has not been verified experimentally at present time, and is not based on biological concepts. Other scenarios explain the observed regular patterns using mechanical models based on elasticity theory: Newell et al. (2008) take a continuous limit to get partial differential equations of the von Karman type where the auxin flux is introduced in stress tensors. This model permits to get regular patterns, and to approach the interplay of mechanics and biochemistry. However, the meristem usually contains only a limited number of cells, and one should explain some aspects of plant morphogenesis using discrete models. We hence proposed a way of coupling the auxin flux model (2) with a potential, which might model mechanical forces, to get the o.d.e. (29). Simulations indicate that the $l$-stable equilibrium configurations are more regular, see Fig. 5. One can also check that the o.d.e (2) is the mean-field limit of a particle system which might model the stochastic movement of auxin molecules (Ethier and Kurtz 1986). There are two among many possibilities, and much remains to be done in this fascinating research field.

Acknowledgments This work was supported by the University of Fribourg, and by the SystemsX "Plant growth in changing environments" project funding. Many thanks to D. Kierzkowski and C. Kuhlemeier for providing us the picture given in Fig. 1 and to Aleš Janka for its help in Matlab programming. We are very grateful to Didier Reinhardt for giving us the opportunity to learn parts of the actual knowledge on the role of the auxin flux in plant patterning.

\section{Appendix: A}

\section{A.1 Proof of the existence of the solution (Proposition 1)}

In the following, we use the notation $\dot{\boldsymbol{a}}$ instead of $\frac{\mathrm{d} \boldsymbol{a}}{\mathrm{d} t}$.

System (2) can be written as

$$
\dot{a}_{i}=D \sum_{k \sim i} a_{k}+T \sum_{k \sim i}\left(\frac{a_{k}}{\kappa+\sum_{j \sim k} a_{j}}-\frac{a_{k}}{\kappa+\sum_{j \sim i} a_{j}}-\frac{D}{T}\right) a_{i}
$$

Let $\boldsymbol{a}$ be a solution of (30) with $\boldsymbol{a}(0) \in \mathbb{R}_{\geq 0}^{L}$.

We say that the function $f: \mathbb{R}_{+} \rightarrow \overrightarrow{\mathbb{R}}$ is instantaneously positive (i.p.) if there exists $\delta>0$ so that $f$ is strictly positive over $(0, \delta)$. If $f(0)>0$ and $f$ is continuous to the right at 0 , then $f$ is i.p.. It is also clear that if $f$ admits a strictly positive right-hand derivative at 0 , then it is i.p..

Let $U$ be the open set $U=\left\{\boldsymbol{x}=\left(x_{1}, x_{2}, \ldots, x_{L}\right) \in \mathbb{R}^{L} ;-\frac{\kappa}{2 L}<x_{i}\right\}$. Since the right-hand member of (30) is continuous over $U$, the general theory of o.d.e.'s provides the existence of a solution defined over a maximal interval $0 \in J^{+} \subset \mathbb{R}_{+}$for any initial condition $\boldsymbol{a}(0) \in U$. Moreover, the solution is unique because the right-hand member of (30) locally lipschitzian. Set for convenience 


$$
\begin{aligned}
& h_{i}(t)=D \sum_{k \sim i} a_{k}(t) \quad \text { and } \\
& g_{i}(t)=T \sum_{k \sim i}\left(\frac{a_{k}(t)}{\kappa+\sum_{j \sim k} a_{j}(t)}-\frac{a_{k}(t)}{\kappa+\sum_{j \sim i} a_{j}(t)}-\frac{D}{T}\right) .
\end{aligned}
$$

The variation of constants formula allows us to write, $\forall t \in J^{+}$,

$$
a_{i}(t)=a_{i}(0) e^{\int_{0}^{t} g_{i}(s) d s}+\int_{0}^{t} h_{i}(u) e^{-\int_{u}^{t} g_{i}(v) d v} d u
$$

Since $a_{i}(0) \geq 0$, the first term in (31) is non-negative. Moreover if $a_{k}(t)$ is i.p. for some $k \sim i$, then according to (31), the same property holds for $a_{i}(t)$. In particular, if $a_{k}(0)>0$ for some $k \sim i$, then by continuity $a_{k}(t)$ is i.p. and thus also $a_{i}(t)$.

The case $D>0$ :

Clearly, if $\boldsymbol{a}(0)=\mathbf{0}$, then the unique solution is identically 0 . Otherwise, there exists $1 \leq i_{0} \leq L$ with $a_{i_{0}}(0)>0$ and $\forall j \sim i_{0}, a_{j}(t)$ is i.p.. Since our graph is supposed to be connected, every $i$ admits a neighbour $k \sim i$ with $a_{k}(t)$ i.p.. Hence, $a_{i}(t)$ is i.p. $\forall i, 1 \leq i \leq L$.

The preceding arguments show that for any initial condition $\boldsymbol{a}(0) \in \mathbb{R}_{\geq 0}^{L} \subset U$, all components of the solution of (30) are i.p.. Let us suppose that one of them admits the value 0 in $J^{+}$. Since all components are continuous and their number is finite, there exists a first time $t_{0}>0$ for which at least one component $a_{i_{0}}\left(t_{0}\right)=0$ and all of them are strictly positive over $\left(0, t_{0}\right)$. According to (31), we have

$$
a_{i_{0}}\left(t_{0}\right)=0=a_{i}(0) e^{\int_{0}^{t_{0}} g_{i}(s) d s}+\int_{0}^{t_{0}} h_{i}(u) e^{-\int_{u}^{t_{0}} g_{i}(v) d v} d u .
$$

Clearly $h_{i}(t)>0$ over $J^{+} \backslash\{0\}$ and since the first term is non-negative, we conclude to $a_{i_{0}}\left(t_{0}\right)>0$, a contradiction. Therefore all $a_{i}(t)$ are strictly positive over $J^{+} \backslash\{0\}$.

The case $D=0$ : If $a_{i}(0)>0, a_{i}(t)$ is i.p. by continuity. In that case $a_{i}(t)=$ $a_{i}(0) e^{\int_{0}^{t} g_{i}(s) d s}>0$ over $J^{+}$. If $a_{i}(0)=0$, the homogeneous equation for $a_{i}(t)$ admits only the zero solution, and we remove the related $i$ th component from (30).

The preceding modification implies that, in both cases, solution of (30) have strictly positive components over $J^{+} \backslash\{0\}$. We also proved that $\forall t \in J^{+}$we have:

$$
\sum_{1 \leq i \leq L} a_{i}(t)=\sum_{1 \leq i \leq L} a_{i}(0)
$$

As a consequence the solution of (30) is bounded and thus the unique solution of our problem is defined over $J^{+}=[0,+\infty)$. 
We easily check that the system $\dot{\boldsymbol{a}}=f(\boldsymbol{a})$ is conservative, as

$$
\begin{aligned}
\sum_{i} \dot{a}_{i}(t) & =D \sum_{i} \sum_{k \sim i}\left(a_{k}-a_{i}\right)+T \sum_{i} \sum_{k \sim i} a_{k} a_{i}\left(\frac{N_{i}-N_{k}}{N_{k} N_{i}}\right) \\
& =D \sum_{i}\left(d_{i} a_{i}-d_{i} a_{i}\right)+\frac{1}{2} T \sum_{i} \sum_{k \sim i} \frac{a_{k} a_{i}}{N_{k} N_{i}}\left(\left(N_{i}-N_{k}\right)+\left(N_{k}-N_{i}\right)\right)=0
\end{aligned}
$$

where $N_{k}=\kappa+\sum_{j \sim k} a_{k}$, and where $d_{i}$ is the degree of $i$ (that is the number of neighbours of $i$ ).

A.2 Proof of the Jacobian matrix in the irreducible case (Proposition 3)

We first give the Jacobian, for an arbitrary $\boldsymbol{a}$. We have

$$
\frac{\partial f_{i}(\boldsymbol{a})}{\partial a_{j}}=\frac{a_{i}}{N_{j}}-\frac{a_{i}}{N_{i}}+\sum_{k \sim i} \frac{a_{i} a_{k}}{N_{i}^{2}}-\sum_{k \sim i, k \sim j} a_{k} \frac{a_{i}}{N_{k}^{2}},
$$

(where the last term is due to the triangles in the graph) when $j \sim i$, that is, $i$ and $j$ are nearest neighbours. When $i=j$, one gets

$$
\frac{\partial f_{i}(\boldsymbol{a})}{\partial a_{i}}=\sum_{k \sim i} \frac{a_{k}}{N_{k}}-a_{i} \sum_{k \sim i} \frac{a_{k}}{N_{k}^{2}}-\frac{\sum_{k \sim i} a_{k}}{N_{i}} .
$$

The remaining non-vanishing partial derivatives correspond to nodes $j$ located at distance 2 of $i$ in the graph, that is, to nodes $j$ such that $j \sim k$ for some $k \sim i, j \neq i$ but $i \neq j$. Then

$$
\frac{\partial f_{i}(\boldsymbol{a})}{\partial a_{j}}=-\sum_{j \sim k, k \sim i} \frac{a_{i} a_{k}}{N_{k}^{2}} .
$$

When $N_{i}=N, \forall i$, these expressions simplify to

$$
\frac{\partial f_{i}(\boldsymbol{a})}{\partial a_{j}}=\sum_{k \sim i} \frac{a_{i} a_{k}}{N_{i}^{2}}=\frac{N-\kappa}{N^{2}} a_{i}-\frac{a_{i}}{N^{2}} \sum_{k \sim i, k \sim j} a_{k} .
$$

If $j \sim i$,

$$
\frac{\partial f_{i}(\boldsymbol{a})}{\partial a_{i}}=-\frac{N-\kappa}{N^{2}} a_{i},
$$

and

$$
\frac{\partial f_{i}(\boldsymbol{a})}{\partial a_{j}}=-\sum_{k \sim i, k \sim j} \frac{a_{i} a_{k}}{N_{k}^{2}}=-\frac{a_{i}}{N^{2}} \sum_{k \sim i, k \sim j} a_{k},
$$

if $j \sim k$ for some $k \sim i, j \neq i$ but $i \not j$. 
Consider the sub-matrix $\boldsymbol{M}$ given by $\boldsymbol{M}=\left(\partial f_{i}(\boldsymbol{a}) / \partial a_{j}\right)_{j \sim i}$. Let $\operatorname{diag}(\boldsymbol{a})$ be the diagonal matrix whose diagonal elements are given by $\boldsymbol{a}$. The perturbation associated with the triangles contained in the graph is represented by the term $-\frac{a_{i}}{N^{2}} \sum_{k \sim i, k \sim j} a_{k}$ in $\frac{\partial f_{i}(\boldsymbol{a})}{\partial a_{j}}$ for $j \sim i$, and the related matrix is given by

$$
\begin{aligned}
\left(-\frac{a_{i}}{N^{2}} \sum_{k \sim i, k \sim j} a_{k}\right) \gamma_{i j} & =\left(-\frac{a_{i}}{N^{2}} \sum_{k} \gamma_{i k} a_{k} \gamma_{k j}\right) \gamma_{i j} \\
& =\left(-\frac{1}{N^{2}}(\operatorname{diag}(\boldsymbol{a}) \boldsymbol{\Gamma} \operatorname{diag}(\boldsymbol{a}) \boldsymbol{\Gamma}-\operatorname{diag}(\operatorname{diag}(\boldsymbol{a}) \boldsymbol{\Gamma} \operatorname{diag}(\boldsymbol{a}) \boldsymbol{\Gamma}))_{i j}\right) \gamma_{i j} \\
& =\left(-\frac{1}{N^{2}}(\operatorname{diag}(\boldsymbol{a}) \boldsymbol{\Gamma} \operatorname{diag}(\boldsymbol{a}) \boldsymbol{\Gamma})_{i j}+\frac{N-\kappa}{N^{2}} \operatorname{diag}(\boldsymbol{a})_{i j}\right) \gamma_{i j} .
\end{aligned}
$$

The matrix $\boldsymbol{M}$ is now given by

$$
\boldsymbol{M}=\frac{\operatorname{diag}(\boldsymbol{a})}{N^{2}}(N-\kappa)(\boldsymbol{\Gamma}-\mathbf{i d})-\frac{1}{N^{2}}(\operatorname{diag}(\boldsymbol{a}) \boldsymbol{\Gamma} \operatorname{diag}(\boldsymbol{a}) \boldsymbol{\Gamma}-(N-\kappa) \operatorname{diag}(\boldsymbol{a})) \circ \boldsymbol{\Gamma},
$$

where $\circ$ represents the matrix Hadamard product, i.e. the multiplication component by component.

Similarly, the perturbation of $\boldsymbol{M}$ by $\left(\frac{\partial f_{i}(\boldsymbol{a})}{\partial a_{j}}\right)_{i \sim k, k \sim j, i \ngtr j, i \neq j}$ can be written as

$$
\begin{gathered}
\left(-\frac{a_{i}}{N^{2}} \sum_{\substack{k \sim i, k \sim j, i \ngtr j, i \neq j}} a_{k}\right) \gamma_{i j}=\left(-\frac{a_{i}}{N^{2}} \sum_{k} \gamma_{i k} a_{k} \gamma_{k j}\right)\left(1-\gamma_{i j}-\mathbf{i d}_{i j}\right) \\
=\left(-\frac{1}{N^{2}}(\operatorname{diag}(\boldsymbol{a}) \boldsymbol{\Gamma} \operatorname{diag}(\boldsymbol{a}) \boldsymbol{\Gamma})_{i j}+\frac{N-\kappa}{N^{2}} \operatorname{diag}(\boldsymbol{a})_{i j}\right)\left(1-\gamma_{i j}-\mathbf{i d}_{i j}\right) .
\end{gathered}
$$

The related Jacobian is thus given by $\boldsymbol{M}+\left(\frac{\partial f_{i}(\boldsymbol{a})}{\partial a_{j}}\right)_{i \sim k, k \sim j, i \chi j, i \neq j}$, that is

$$
\begin{aligned}
\mathrm{d} f(\boldsymbol{a})= & \frac{\operatorname{diag}(\boldsymbol{a})}{N^{2}}(N-\kappa)(\boldsymbol{\Gamma}-\mathbf{i d})-\frac{1}{N^{2}}(\operatorname{diag}(\boldsymbol{a}) \boldsymbol{\Gamma} \operatorname{diag}(\boldsymbol{a}) \boldsymbol{\Gamma}-(N-\kappa) \operatorname{diag}(\boldsymbol{a})) \circ \boldsymbol{\Gamma} \\
& -\frac{1}{N^{2}}(\operatorname{diag}(\boldsymbol{a}) \boldsymbol{\Gamma} \operatorname{diag}(\boldsymbol{a}) \boldsymbol{\Gamma}-(N-\kappa) \operatorname{diag}(\boldsymbol{a})) \circ(\mathbf{1}-\boldsymbol{\Gamma}-\mathbf{i d}) \\
= & \frac{\operatorname{diag}(\boldsymbol{a})}{N^{2}}(N-\kappa)(\boldsymbol{\Gamma}-\mathbf{i d})-\frac{1}{N^{2}}(\operatorname{diag}(\boldsymbol{a}) \boldsymbol{\Gamma} \operatorname{diag}(\boldsymbol{a}) \boldsymbol{\Gamma}-(N-\kappa) \operatorname{diag}(\boldsymbol{a})) \circ(\mathbf{1}-\mathbf{i d}) \\
= & \frac{\operatorname{diag}(\boldsymbol{a})}{N^{2}}(N-\kappa)(\boldsymbol{\Gamma}-\mathbf{i d})-\frac{1}{N^{2}}(\operatorname{diag}(\boldsymbol{a}) \boldsymbol{\Gamma} \operatorname{diag}(\boldsymbol{a}) \boldsymbol{\Gamma}-(N-\kappa) \operatorname{diag}(\boldsymbol{a})),
\end{aligned}
$$


where $\mathbf{1}$ is the matrix composed of ones only. The last equality is a consequence of the fact that the $\operatorname{diagonal}$ of $\operatorname{diag}(\boldsymbol{a}) \boldsymbol{\Gamma} \operatorname{diag}(\boldsymbol{a}) \boldsymbol{\Gamma}-(N-\kappa) \operatorname{diag}(\boldsymbol{a})$ vanishes. Hence,

$$
\mathrm{d} f(\boldsymbol{a})=\frac{\operatorname{diag}(\boldsymbol{a}) \boldsymbol{\Gamma}}{N^{2}}((N-\kappa) \mathbf{i d}-\operatorname{diag}(\boldsymbol{a}) \boldsymbol{\Gamma})=\frac{\operatorname{diag}(\boldsymbol{a}) \boldsymbol{\Gamma}}{N^{2}}(c \mathbf{i d}-\operatorname{diag}(\boldsymbol{a}) \boldsymbol{\Gamma}),
$$

proving the result.

\section{A.3 Proof of the spectrum in the reducible case (Proposition 4)}

Set $I=\left\{i \in \Lambda: a_{i}=0\right\}$, and consider the subgraphs $\gamma_{p}$ of $G$ induced by the nodes of $J=\Lambda \backslash I$, with $\gamma_{p}=\left(\Lambda_{p}, E_{p}\right), 1 \leq p \leq P$. For any related equilibrium point $\boldsymbol{a}$, the restrictions $\left.\boldsymbol{a}\right|_{\gamma_{p}}$ satisfy the linear systems $\left.\boldsymbol{\Gamma}_{\gamma_{p}} \boldsymbol{a}\right|_{\gamma_{p}}=\left.c_{\gamma_{p}} \mathbf{1}\right|_{\gamma_{p}}$. Set $N_{\gamma_{p}}=c_{\gamma_{p}}+\kappa$. Equations (32)-(34) allow the computation of the entries of the Jacobian matrix. By first looking at the diagonal entries, for $i \in \Lambda_{p}$, one has

$$
\frac{\partial f_{i}(\boldsymbol{a})}{\partial a_{i}}=-a_{i} \frac{N_{\gamma_{p}}-\kappa}{N_{\gamma_{p}}^{2}}
$$

providing the diagonal entry of the Jacobian of $\left.f\right|_{\gamma_{p}}\left(\left.\boldsymbol{a}\right|_{\gamma_{p}}\right)$. When $i \notin \Lambda_{p}$, a similar computation yields

$$
\frac{\partial f_{i}(\boldsymbol{a})}{\partial a_{i}}=\sum_{k \sim i} \frac{a_{k}}{N_{k}}-\frac{N_{i}-\kappa}{N_{i}} .
$$

We then compute the entries $(i, j)$ for $j \sim i, i, j \in \Lambda_{p}$ and $1 \leq p \leq P$ :

$$
\frac{\partial f_{i}(\boldsymbol{a})}{\partial a_{j}}=a_{i} \frac{N_{\gamma_{p}}-\kappa}{N_{\gamma_{p}}^{2}}-\sum_{k \sim i, k \sim j} a_{k} \frac{a_{i}}{N_{k}^{2}}=a_{i} \frac{N_{\gamma_{p}}-\kappa}{N_{\gamma_{p}}^{2}}-\sum_{k \sim i, k \sim j, k \in \Lambda_{p}} \frac{a_{k} a_{i}}{N_{\gamma_{p}}^{2}},
$$

This corresponds to the $(i, j)$ entry of the Jacobian of $\left.f\right|_{\gamma_{p}}\left(\left.\boldsymbol{a}\right|_{\gamma_{p}}\right)$. Similarly, when $i \in \Lambda_{p}$ for some $p$ and $j \notin \Lambda_{p}$

$$
\frac{\partial f_{i}(\boldsymbol{a})}{\partial a_{j}}=\frac{a_{i}}{N_{j}}-\frac{a_{i}}{N_{\gamma_{p}}}+\sum_{k \sim i} \frac{a_{i} a_{k}}{N_{\gamma_{p}}^{2}}-\sum_{\substack{k \sim i, k \sim j, k \in \Lambda_{p}}} \frac{a_{k} a_{i}}{N_{\gamma_{p}}^{2}}=\frac{a_{i}}{N_{j}}-a_{i} \frac{\kappa}{N_{\gamma_{p}}^{2}}-\frac{a_{i}}{N_{\gamma_{p}}^{2}} \sum_{\substack{k \sim i, k \sim j, k \in \Lambda_{p}}} a_{k} .
$$

Finally, when $i, j \notin \cup_{p} \Lambda_{p}$, or equivalently when both $i$ and $j$ belongs to $I$

$$
\frac{\partial f_{i}(\boldsymbol{a})}{\partial a_{j}}=0
$$


We next consider $(i, j)$ entries where $j$ is at a distance 2 of $i$ in the graph $G$, that is when $j$ is such that $j \sim k$ for some $k \sim i, j \neq i$ and $j \neq i$. For $i, j, k \in \Lambda_{p}$

$$
\frac{\partial f_{i}(\boldsymbol{a})}{\partial a_{j}}=-a_{i} \sum_{j \sim k, k \sim i} \frac{a_{k}}{N_{\gamma_{p}}^{2}}
$$

This is the $(i, j)$ entry of the Jacobian of $\left.f\right|_{\gamma_{p}}\left(\left.\boldsymbol{a}\right|_{\gamma_{p}}\right)$.

Similarly, for $i, k \in \Lambda_{p}, j \notin \Lambda_{p}(\Rightarrow j \in I)$

$$
\frac{\partial f_{i}(\boldsymbol{a})}{\partial a_{j}}=-a_{i} \sum_{j \sim k, k \sim i} \frac{a_{k}}{N_{\gamma_{p}}^{2}} .
$$

Next, for $i$ or $k \notin \Lambda_{p}, \forall j \in \Lambda$

$$
\frac{\partial f_{i}(\boldsymbol{a})}{\partial a_{j}}=0
$$

Permuting conveniently the indices, the Jacobian $\mathrm{d} f(\boldsymbol{a})$ can be written as

$$
\mathrm{d} f(\boldsymbol{a})=\left(\begin{array}{cc}
\boldsymbol{d}_{n} & \mathbf{0} \\
* & \mathrm{~d} f^{\gamma}
\end{array}\right)
$$

where $\boldsymbol{d}_{n}$ is a diagonal matrix $n \times n$ with entries $\lambda_{i}:=\sum_{k \sim i} \frac{a_{k}}{N_{k}}-\frac{N_{i}-\kappa}{N_{i}}$, for $i \in I$, and $\mathrm{d} f^{\gamma}$ is a block diagonal matrix, each block being equal to the Jacobian of $f$ restricted on each subgraph $\gamma_{p}$. The permutation allows grouping of all indices $i \in I$ in the same block, and all indices related to the subgraphs $\gamma_{p}$ are also arranged together. It follows that the spectrum of $\mathrm{d} f(\boldsymbol{a})$ is :

$$
\operatorname{spec}(\mathrm{d} f(\boldsymbol{a}))=\bigcup_{p=1}^{P} \operatorname{spec}\left(\left.\mathrm{d} f\right|_{\gamma_{p}}\left(\left.\boldsymbol{a}\right|_{\gamma_{p}}\right)\right) \cup\left\{\sum_{k \sim i} \frac{a_{k}}{N_{k}}-\frac{N_{i}-\kappa}{N_{i}}, i \in I\right\} .
$$

\section{A.4 Proof of Proposition 5}

To prove the Proposition 5, we will use the following Proposition, see Gabriel et al. (1989).

Proposition 9 Let $f: \mathbb{R}_{+} \rightarrow \mathbb{R}$ be twice differentiable and bounded together with $\ddot{f}$. If, as $n \rightarrow+\infty, t_{n} \uparrow+\infty$ and $f\left(t_{n}\right) \rightarrow \liminf _{t \rightarrow+\infty} f(t)\left(\right.$ or $f\left(t_{n}\right) \rightarrow$ $\left.\lim \sup _{t \rightarrow+\infty} f(t)\right)$, then $\dot{f}\left(t_{n}\right) \rightarrow 0$.

Proof If $a_{k}(0)=0$ for all $1 \leq k \leq L$, then the unique solution is identically zero. Otherwise $\sum_{k=1}^{L} a_{k}(0)>0$. Let us suppose that for some $i \in\{1, \ldots, L\}$,

$$
\liminf _{t \rightarrow+\infty} a_{i}(t)=0
$$


Let us introduce the notation $\underline{a}_{i}=\liminf \operatorname{in}_{t \rightarrow+\infty} a_{i}(t)$. Since $a_{i}(t)$ is bounded together with its second derivative, the preceding proposition applies and for any sequence $t_{n} \uparrow+\infty$ such that $a_{i}\left(t_{n}\right) \rightarrow \underline{a}_{i}$, we have $\dot{a}_{i}\left(t_{n}\right) \rightarrow 0$ as $n \rightarrow+\infty$. Every $a_{k}\left(t_{n}\right)$ being bounded in the right-hand member of the equation for $\dot{a}_{i}\left(t_{n}\right)$, we conclude that $\lim _{n \rightarrow+\infty} D \sum_{k \sim i} a_{k}\left(t_{n}\right)=0$. The non-negativity of each $a_{k}\left(t_{n}\right)$ entails $\lim _{n \rightarrow+\infty} a_{k}\left(t_{n}\right)=0=\underline{a}_{k}$ for every $k \sim i$. According to the above proposition, $\lim _{n \rightarrow+\infty} \dot{a}_{k}\left(t_{n}\right)=0$ for every $k \sim i$ and since the graph is connected, repeating the same argument provides $\lim _{n \rightarrow+\infty} \dot{a}_{j}\left(t_{n}\right)=0$ for every $j \in\{1, \ldots, L\}$. Thus $0=\lim _{n \rightarrow+\infty} \sum_{1 \leq j \leq L} a_{j}\left(t_{n}\right)=\sum_{k=1}^{L} a_{k}(0)>0$, a contradiction.

\section{References}

Adler I (1974) A model of contact pressure in phyllotaxis. J Theor Biol 1:1-79

Atela P, Golé C, Hotton C (2002) A dynamical system for plant pattern formation. J Nonlinear Sci 12:641676

Barbier de Reuille P, Bohn-Courseau I, Ljung K, Morin H, Carraro N, Godin C, Traas J (2006) Computer simulations reveal properties of the cell-cell signaling network at the shoot apex in Arabidopsis. Proc Natl Acad Sci USA 103:1627-1632

Benaïm M (1997) Vertex-reinforced random walks and a conjecture of pemantle. Ann Prob 25:361-392

Benaïm M, Tarrès P (2008) Dynamics of vertex-reinforced random walks. ArXiv e-prints 0809.2739v3

Boudaoud A (2010) An introduction to the mechanics of morphogenesis for plant biologists. Trends Plant Sci 15:353-360

Diaconis P, Stroock D (1991) Geometric bounds for Eigenvalues of Markov chains. Ann Appl Probab $1: 36-61$

Douady S, Couder Y (1996) Phyllotaxis as a dynamical self organizing process (Part I, II, III). J Theor Biol 178:255-312

Dumais J (2007) Can mechanics control pattern in plants? Curr Opin Plant Biol 10:58-62

Dumais J, Steele C (2000) New evidence for the role of mechanical forces in the shoot apex meristem. J Plant Growth Regul 19:7-18

Ethier SN, Kurtz TG (1986) Markov processes: characterization and convergence. Wiley Ser Probab Math Stat

Gabriel JP, Hanisch H, Hirsch W (1988-1989) Prepatency and sexuality of parasitic worms: the hermaphroditic case, Anno 3, vol 4. Atti del colloquio di matematica, Edizione Cerfim Locarno

Green P (1980) Organogenesis_a biophysical view. Annu Rev Plant Physiol 31:51-82

Hamant O, Heisler MG, Jönsson H, Krupinski P, Uytterwaal M, Bokov P, Corson F, Sahlin P, Boudaoud A, Meyerowitz E, Couder Y, Traas J (2008) Developmental patterning by mechanical signals in Arabidopsis. Science 322:1650-1655

Hamant O, Traas J (2009) The mechanics behind plant development. New Phytol 185:369-385

Heisler MG, Jönsson H (2006) Modeling auxin transport and plant development. J Plant Growth Regul 25:302-312

Hofmeister W (1868) Handbuch der Physiologischen Botanik: Allgemeine Morphologie der Gewächse. Engelmann, Leipzig

Jönsson H, Heisler MG, Shapiro BE, Mjolsness E, Meyerowitz EM (2006) An auxin-driven polarized transport model for phyllotaxis. Proc Natl Acad Sci USA 103:1633-1638

Kunz M (1995) Some analytical results about two physical models of phyllotaxis. Commun Math Phys 169:261-295

LaSalle JP (1960) Some extensions of Liapunov's second method. IRE Trans CT 7:520-527

Levitov LS (1991) Energetics approach to phyllotaxis. Europhys Lett 14:533-539

Meinhardt H (1982) Models of biological pattern formation. Academic Press, London

Mjolsness E (2006) The growth and development of some recent plant models: a viewpoint. J Plant Growth Regul 25:270-277

Motzkin T, Straus G (1965) Maxima for graphs a new proof of a theorem of Turán. Can J Math 17:533-540

Newell A, Shipman P (2005) Plant and fibonacci. J Stat Phys 121:937-968 
Newell AC, Shipman PD, Sun Z (2008) Phyllotaxis: cooperation and competition between mechanical and biochemical processes. J Theor Biol 251:421-439

Norris JR (1997) Markov chains. Camb Ser Stat Probab Math

Pemantle R (1992) Vertex-reinforced random walk. Probab Theory Relat Fields 92:117-136

Reinhardt D (2005) Phyllotaxis - a new chapter in an old tale about beauty and magic numbers. Curr Opin Plant Biol 8:487-493

Reinhardt D, Mandel T, Kuhlemeier C (2000) Auxin regulates the initiation and radial position of lateral organs. Plant Cell 12:501-518

Reinhardt D, Pesce E, Stieger P, Mandel T, Baltensperger K, Bennett M, Traas J, Friml J, Kuhlemeier C (2003) Regulation of phyllotaxis by polar auxin transport. Nature 426:255-260

Sahlin P, Söderberg B, Jönsson H (2009) Regulated transport as a mechanism for pattern generation: capabilities for phyllotaxis and beyond. J Theor Biol 258:60-70

Shipman PD, Newell AC (2005) Polygonal plantform and phyllotaxis on plants. J Theor Biol 236:154-197

Smith RS, Guyomarch's S, Mandel T, Reinhardt D, Kuhlemeier C et al (2006) A plausible model of phyllotaxis. Proc Natl Acad Sci USA 103:1301-1306

Thornley J (1975) Phyllotaxis I. A mechanistic model. Ann Botany 39:491-507

Turing A (1952) The chemical basis of morphogenesis. Philos Trans R Soc Lond 237:37-72

Waller D (1977) Optimisation of quadratic forms associated with graphs. Glasgow Math J 18:79-85 